\title{
The dispersion-brightness relation for fast radio bursts from a wide-field survey
}

R. M. Shannon ${ }^{1,2,3}$, J.-P. Macquart ${ }^{2,4}$, K. W. Bannister ${ }^{3}$, R. D.Ekers ${ }^{2,3}$, C. W. James ${ }^{2,4}$, S. Osłowski ${ }^{1}$, H. Qiu ${ }^{5,3,4}$, M. Sammons ${ }^{2}$, A. W. Hotan ${ }^{6}$, M. A. Voronkov ${ }^{3}$, R. J. Beresford ${ }^{3}$, M. Brothers ${ }^{3}$, A. J. Brown ${ }^{3}$, J. D. Bunton ${ }^{3}$, A. P. Chippendale ${ }^{3}$, C. Haskins ${ }^{6}$, M. Leach ${ }^{3}$, M. Marquarding ${ }^{3}$, D. McConnell ${ }^{3}$, M. A. Pilawa ${ }^{3}$, E. M. Sadler ${ }^{5,4}$, E. R. Troup ${ }^{3}$, J. Tuthill ${ }^{3}$, M. T. Whiting ${ }^{3}$, J. R.

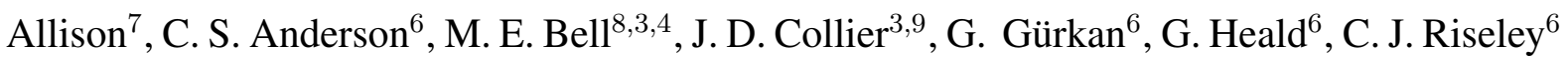

${ }^{1}$ Centre for Astrophysics and Supercomputing, Swinburne University of Technology, Mail H30, PO Box 218, Hawthorn, VIC 3122, Australia.

${ }^{2}$ International Centre for Radio Astronomy Research, Curtin Institute of Radio Astronomy, Curtin University, Perth, WA 6845, Australia

${ }^{3}$ CSIRO Astronomy and Space Science, Australia Telescope National Facility, P.O. Box 76, Epping, NSW 1710, Australia

${ }^{4}$ ARC Centre of Excellence for All-Sky Astrophysics (CAASTRO)

${ }^{5}$ Sydney Institute for Astrophysics, School of Physics, University of Sydney, Sydney, New South Wales 2006, Australia.

${ }^{6}$ CSIRO Astronomy and Space Science, Australia Telescope National Facility, PO Box 1130, Bentley WA 6102, Australia

${ }^{7}$ Sub-Dept. of Astrophysics, Department of Physics, University of Oxford, Denys Wilkinson Building, Keble Rd., Oxford, OX1 3RH, UK

${ }^{8}$ School of Mathematics and Physical Sciences, University of Technology Sydney, 15 Broadway, Ultimo NSW 2007 
${ }^{9}$ School of Computing, Engineering, and Mathematics, Western Sydney University, Locked Bag 1797, Penrith, NSW 2751, Australia 
Despite considerable efforts spanning the last decade, only 34 fast radio bursts (FRBs) have been reported ${ }^{1,2}$. Attempts to understand the population as a whole have been hindered by highly heterogeneous searches, conducted with telescopes with different sensitivities, at a range of radio frequencies, in environments corrupted by different, and oftentimes hostile levels of radio-frequency interference. The searches have been further complicated by uncertain burst positions and brightnesses, a consequence of the transient nature of the sources, and the poor angular resolution of the detecting instruments. The discovery of repeating bursts from one source ${ }^{3}$, and its subsequent localization ${ }^{4}$ to a dwarf galaxy at a distance of 3.7 billion light years, confirmed that the population is located at cosmological distances. However the nature of the emission remains elusive. Here we report a well-controlled radio survey for these bursts. We found 20 , none of which repeated during follow-up observations ranging from 185 Tto $1097 \mathbf{~ h r}$. The sample includes both the nearest and the most energetic bursts detected to date. The survey has demonstrated that the high-fluence bursts are the nearby analogues of the more distant events found in higher sensitivity, narrower-field surveys 5 .

Since the beginning of 2017 , we have been surveying for fast radio bursts using a subset of the Australian Square Kilometre Array Pathfinder ${ }^{[6}$ (ASKAP), a radio-telescope array comprising 36 antennas currently being commissioned. Each antenna is equipped with a phasedarray-feed receiver (PAF) sensitive to $30 \mathrm{deg}^{2}$ on the sky at the prime focus of a 12-m antenna. The searches, conducted at a central frequency of $1.3 \mathrm{GHz}$, have employed a fly's-eye configuration, with each of $5-12$ antennas available pointed toward a different area of sky, widening our field of view to target the brightest portion of the burst population. The system setup and search algorithms are identical to those reported previously from ASKAP ${ }^{7}$. The PAFs allow 
full sampling of the focal plane, rendering it possible to measure the burst positions with as little as $10 \times 10 \operatorname{arcmin}$ uncertainty ${ }^{7}(90 \%$ confidence $)$ and the fluence of each burst to better than $20 \%$ accuracy $y^{7}$, in contrast to previous searches where burst positions were unconstrained within the antenna beam pattern, and burst fluences could be uncertain by factors greater 10 . The searches have been conducted at 57 high Galactic latitude pointings $|b|=50 \pm 5 \mathrm{deg}$, removing the need to account for potential bias in the latitude dependence of the rate ${ }^{8}$ and minimizing the contribution of the Galaxy to the electron column density. Observations of these pointings were interleaved with short scans of known pulsar to check system performance (see supplementary section 1). Pointings were revisited a median of 570 times over the course of the survey. In total our survey exposure is $5.1 \times 10^{5} \mathrm{deg}^{2} \mathrm{~h}$ (see supplementary section 1$)$.

We have discovered 20 FRBs in total (including FRB 170107 reported previously ${ }^{7}$ ), the properties of which are listed in Table 1. The electron column densities towards the bursts, expressed in dispersion measure (DM), range from 114 to $998 \mathrm{pc} \mathrm{cm}^{-3}$, The dispersion measures contain contributions from the Milky Way, the host galaxy, and the intergalactic medium. The Galactic DM contributions are likely to be $<60 \mathrm{pc} \mathrm{cm}^{-3}$ for all of our bursts. Assuming a negligible circumburst environment, the host galaxy is likely to have a similar median contribution ${ }^{9}$ (See supplementary section 4). Larger contributions would be possible if the bursts were embedded in dense nebulae such as supernova remnants or in galaxy centres. However, our lowest DM events show that for some objects this contribution does not exceed $50-120 \mathrm{pc} \mathrm{cm}^{-3}$. We also assume that the intergalactic medium is uniformly distributed and use a standard distanceDM relationship $\frac{10}{10}$ to infer distances from the extragalactic DM component. For the nearest (and hence lowest DM) bursts, larger stochasticity in the intergalactic DM contribution would be expected as it will depend strongly on the host location with the local large scale structure ${ }^{11}$, 
which has a characteristic size scale of $30 \mathrm{Mpc}$. For more distant objects, bursts will propagate through many void and walls and therefore will only have modest $10-20 \%$ scatter ${ }^{11}$. Our sample includes the lowest hitherto reported DM for a burst (FRB 171020), which has a column density excess beyond the Milky Way ${ }^{12}$ of $80 \mathrm{pc} \mathrm{cm}^{-3}$. Given our assumed DM-distance model described in the previous paragraph, the burst originates at a distance of approximately $130 \mathrm{Mpc}(z \approx 0.03)$. This burst has one of the poorest localisations $(30 \times 50 \operatorname{arcmin}$ ellipse, $90 \%$ confidence) in the sample because it was detected in a corner beam. However there is only one galaxy catalogued at a distance $<210 \mathrm{Mpc}(z<0.05)$ within the uncertainty region of the FRB. This is the distorted Sc galaxy PGC 068417, at a distance of $37 \mathrm{Mpc}^{13}(z=0.0087)$. A second galaxy at the edge of the error box, PGC 3094828 has a catalogued redshift of $z=0.0665$, placing it at a distance of $275 \mathrm{Mpc}^{14}$. Low dispersion burst sources such as FRB 171020 have the potential for detailed host-galaxy studies.

The measured fluences range from 34 to $390 \mathrm{Jy} \mathrm{ms}$, with the latter being the highest well-constrained fluence (see supplementary section 4). Above our completeness threshold of a signal-to-noise ratio of 9.5 , which corresponds to a fluence of $26 \mathrm{Jy} \mathrm{ms}(t / 1.26 \mathrm{~ms})^{-1 / 2}$ for a pulse duration matching our time resolution of $1.26 \mathrm{~ms}$, the event rate is $37 \pm 8$ per day over the entire sky ( $1 \sigma$ uncertainty). This is a factor of 200 less than the rate from high Galactic latitude searches with the $64-\mathrm{m}$ Parkes radio telescope ${ }^{5}$. This difference is a consequence of a number of factors including the burst fluence and width distributions, telescope sensitivities, and instrumental selection effects.

All bursts in the sample show strong spectral modulation, as seen in Figure 1. While some, such as FRB 170712, show broad-band structure persisting across half of the band, many 
exhibit power concentrated in narrower structures of a few megahertz bandwidth, with signals absent in large fractions of the band. Others, such as FRB 170906, show strong narrow-band features imposed on broad-band structures. It is unclear if the spectral modulation is intrinsic to the source, caused by diffractive scintillation, or refractive propagation effects such as causticinduced magnification 15 . The Parkes population, comprising 26 bursts detected in the same frequency band as ASKAP, shows less evidence for strong spectral modulation, even at high Galactic latitudes ${ }^{5}$, where Galactic propagation would be expected to impart similar spectral structure to the ASKAP bursts. There are notable counterexamples in the Parkes sample that show spectral modulation: the highest signal-to-noise ratio Parkes detection FRB 18030916, and FRB 150807, which shows spectral modulation on two scales $\frac{17}{17}$. Studies of the bright population in a different frequency range would distinguish between causes for the spectral modulation, because propagation effects are strongly frequency dependent. We averaged the spectra to estimate the global properties of the detections, finding the spectral index of combined spectra of all the bursts to be steep, with $\beta=-1.8 \pm 0.3$ over our observing band (where fluence $E(\nu)$ scales proportional to $E(\nu) \propto \nu^{\beta}$ ) (See supplementary section 2.).

Temporal analyses of the burst profiles are limited by the $1.26 \mathrm{~ms}$ time resolution of our current data sets. All of the bursts are marginally resolved beyond instrumental dispersion smearing, with a median burst full width at half maximum being $3.0 \mathrm{~ms}$. This is comparable to the median burst width of the Parkes sample $e^{18}$. Three bursts (FRBs 180110, 180119, and 180130) show exponential profiles consistent with scatter broadening. The broadening time for FRB 180110 varies with frequency, scaling proportional to $\nu^{-3.5 \pm 0.6}$, consistent with propagation through turbulent plasma ${ }^{19}$. FRBs 180119 and 180130 are too weak to conclusively detect a change in pulse-broadening with frequency. All three additionally show spectral modulation; 
if this is caused by scattering, the presence of both indicates that there is propagation through two distinct scattering regions along the line sigh ${ }^{2021}$.

As the survey frequently revisits the same positions, we can place strong constraints on burst repetition. We find no events at similar dispersion measures exceeding a signal-to-noise ratio of 9 at the DM and the positions of the ASKAP FRBs. Dwell times at these positions range from 8 days to 47 days (see Table 1), and 236 to 1235 visits to the fields. In total 12, 456 hours of observations were conducted in the direction of the detected FRBs, and 61 days of observation of the FRB fields were conducted within \pm 15 days of the FRB detection. For one of the detected bursts, FRB 171019, we conducted follow-up observations with the 64-m Parkes radio telescope 2 and 3 days after the FRB detection. In a total of $1 \mathrm{hr}$ of observing, no pulses were detected at the DM of FRB 171019, above a limiting fluence of $1.5 \mathrm{Jy} \mathrm{ms}$, which is a factor of 150 fainter than the FRB 171019 detection.

The fluence distribution of the ASKAP sample may be indicative of a cosmologically evolving population. The distribution of the ASKAP sample was examined with the $V / V_{\max }$ test statistic ${ }^{22}$, which uses the measured signal to noise ratio $(\mathrm{S} / \mathrm{N})$ for each burst to assess the volume within which each burst was detected, relative to the maximum volume in which it could have been detected in Euclidean space-time. In such a population, distributed homogeneously over a volume small enough that the curvature of the Universe is negligible, the ensemble-averaged value of the statistic $V / V_{\max }$ would be 0.5 . For the bursts in the ASKAP sample above our completeness threshold, we find $\left\langle V / V_{\max }\right\rangle=0.58 \pm 0.07$. This is consistent with being produced by a Euclidean population with $12 \%$ confidence (see supplementary section 3). The one-sided probability was determined by simulations that model a realistic 
burst population with dispersion and widths consistent with the observed ASKAP population, detected by a system with our characteristics. For a pure power-law integral source count distribution $N(>E) \propto E^{\alpha}$, the measured $V / V_{\max }$ value implies $\alpha=-2.1_{-0.5}^{+0.6}(67 \%$ confidence) over the range of fluences probed by ASKAP, evidence for steeper-than-Euclidean fluence distribution ${ }^{23}$.

Comparison of the DM distributions of the ASKAP and Parkes samples show that DM is a distance indicator. The median DM of the ASKAP sample is $441 \mathrm{pc} \mathrm{cm}^{-3}$, which is a factor of two smaller than that of the Parkes sample, $880 \mathrm{pc} \mathrm{cm}^{-3}$. A Kolmogorov-Smirnov test finds that the probability that the two distributions are inconsistent is $99.9 \%$. The difference in DM distributions cannot be explained by the poorer time and frequency resolution of the ASKAP system (See supplementary section 5.). This confirms that there is both a correlation between DM and source fluence and that DM can be used as a proxy for distance. However the difference in the distributions is smaller than would be expected for a non-evolving Euclidean population. For any individual burst, the average fluence decreases with the inverse square of distance. Because Parkes is a factor of approximately 50 more sensitive than ASKAP, it would be sensitive to the same source a factor of $\approx 7$ more distant. As we are comparing the median of the populations detected with Parkes and ASKAP, and not individual sources or standard candles, the ratio of dispersion measures is smaller, a consequence of a broad luminosity function for the population 24 .

Figure 2 shows both the fluence-extragalactic dispersion measure distribution for all published FRBs and the fluence-distance relationship, the latter assuming the model for host DM contribution and extragalactic dispersion described above. The solid black curves are contours 
of constant energy density, calculated assuming pulses are isotropically beamed, and using the global spectral index to correct to the rest frame of the emitter. The blue-dashed lines show the extrapolation of ASKAP FRB fluences to higher distances. Notably, the highest DM event from Parkes ${ }^{25}$, FRB 160102, has an inferred energy comparable to those observed in ASKAP. Based on this extrapolation, the energies of the Parkes bursts overlap those of our sample, and are therefore more distant versions of the ASKAP events. The absence of sources above a spectral energy density of approximately $10^{34} \mathrm{erg} \mathrm{Hz}^{-1}$ for both the Parkes and ASKAP samples is unlikely to be solely due to the frequency and temporal resolution of the data recording systems, so could either represent a dwindling population or an energy cutoff (See supplementary section 5.).

There are also marked differences between the ASKAP and Parkes burst populations, and the repeating FRB 121102. Firstly, the ASKAP and Parkes samples show no evidence for repetition, despite large amounts of follow-up time and dense searches around the time of FRB detections. The repetition rate of the repeater is intermittent, with frequent detections on month-long time scales followed by similar length periods of apparent quiescence $\sqrt{3 \mid 26}$. The absence of repetition enables us to reject the hypothesis that all ASKAP FRBs repeat with the same properties ${ }^{26}$ as FRB 121102 at the $99 \%$ confidence level (See supplementary section 6). Secondly, the population of ASKAP bursts have a steep spectrum. While pulses from the repeating FRB show strong spectral modulation, equally energetic pulses are detected over a frequency range extending from 1.4 to $8 \mathrm{GHz}^{27}$. Furthermore, FRB 121102 is underluminous relative to the remaining bursts, as displayed in Figure 2.

The results presented here build on previously noted differences between the repeating 
and the remaining sources. For example, measurements of Faraday rotation suggest that luminous bursts propagate through dispersing plasma that is nonmagnetised ${ }^{17}$, weakly magnetised ${ }^{20 \mid 28}$, or has highly disordered magnetic fields. Such large Faraday rotations could still be hidden in the unpolarised FRBs but it would be impossible to hide for those with significant polarization. In contrast, the repeating FRB source is found to have Faraday rotation (and hence magnetic field strengths) more than four orders of magnitude larget ${ }^{27}$. We do not currently have the capabilities to measure Faraday rotation with ASKAP in FRB search mode, but expect to upgrade these systems to make the necessary polarimetric observations shortly. We are also commissioning interferometric modes and expect to shortly be able to localize detections to arcsecond accuracy. Unique identification of host galaxies will further distinguish between repeating and non-repeating burst sources. 
1. Lorimer, D. R., Bailes, M., McLaughlin, M. A., Narkevic, D. J. \& Crawford, F. A Bright Millisecond Radio Burst of Extragalactic Origin. Science 318, 777 (2007).

2. Petroff, E. et al. FRBCAT: The Fast Radio Burst Catalogue. Publ.Astron.Soc.Australia 33, e045 (2016)

3. Spitler, L. G. et al. A repeating fast radio burst. Nature 531, 202-205 (2016).

4. Chatterjee, S. et al. A direct localization of a fast radio burst and its host. Nature 541, 58-61 (2017).

5. Champion, D. J. et al. Five new fast radio bursts from the HTRU high-latitude survey at Parkes: first evidence for two-component bursts. Mon. Not. R. Astro. Soc. 460, L30-L34 (2016).

6. McConnell, D. et al. The Australian Square Kilometre Array Pathfinder: Performance of the Boolardy Engineering Test Array. Publ.Astron.Soc.Australia 33, e042 (2016).

7. Bannister, K. W. et al. The Detection of an Extremely Bright Fast Radio Burst in a Phased Array Feed Survey. Astrophys. J. 841, L12 (2017).

8. Macquart, J.-P. \& Johnston, S. On the paucity of fast radio bursts at low Galactic latitudes. Mon. Not. R. Astro. Soc. 451, 3278-3286 (2015).

9. Xu, J. \& Han, J. L. Extragalactic dispersion measures of fast radio bursts. Research in Astronomy and Astrophysics 15, 1629 (2015).

10. Inoue, S. Probing the cosmic reionization history and local environment of gamma-ray bursts through radio dispersion. Mon. Not. R. Astro. Soc. 348, 999-1008 (2004). 
11. McQuinn, M. Locating the "Missing" Baryons with Extragalactic Dispersion Measure Estimates. Astrophys. J. 780, L33 (2014).

12. Yao, J. M., Manchester, R. N. \& Wang, N. A New Electron-density Model for Estimation of Pulsar and FRB Distances. Astrophys. J. 835, 29 (2017).

13. Meyer, M. J. et al. The HIPASS catalogue - I. Data presentation. Mon. Not. R. Astro. Soc. 350, 1195-1209 (2004).

14. Jones, D. H. et al. The 6dF Galaxy Survey: Final redshift release (DR3) and southern large-scale structures. MNRAS 399, 683-698 (2009).

15. Cordes, J. M. et al. Lensing of Fast Radio Bursts by Plasma Structures in Host Galaxies. Astrophys. J. 842, 35 (2017).

16. Oslowski, S. et al. Real-time detection of an extremely high signal-to-noise ratio fast radio burst during observations of PSR J2124-3358. The Astronomer's Telegram 11385 (2018).

17. Ravi, V. et al. The magnetic field and turbulence of the cosmic web measured using a brilliant fast radio burst. Science 354, 1249-1252 (2016).

18. Ravi, V. The observed properties of Fast Radio Bursts. ArXiv e-prints (2017).

19. Lambert, H. C. \& Rickett, B. J. On the Theory of Pulse Propagation and Two-Frequency Field Statistics in Irregular Interstellar Plasmas. Astrophys. J. 517, 299-317 (1999).

20. Masui, K. et al. Dense magnetized plasma associated with a fast radio burst. Nature 528, $523-525(2015)$. 
21. Farah, W. et al. FRB microstructure revealed by the real-time detection of FRB170827. Mon. Not. R. Astro. Soc. (2018).

22. Oppermann, N., Connor, L. D. \& Pen, U.-L. The Euclidean distribution of fast radio bursts. Mon. Not. R. Astro. Soc. 461, 984-987 (2016).

23. Macquart, J.-P. \& Ekers, R. D. Fast radio burst event rate counts - I. Interpreting the observations. Mon. Not. R. Astro. Soc. 474, 1900-1908 (2018).

24. von Hoerner, S. Radio Source Counts and Cosmology. Astrophys. J. 186, 741-766 (1973).

25. Bhandari, S. et al. The SUrvey for Pulsars and Extragalactic Radio Bursts - II. New FRB discoveries and their follow-up. Mon. Not. R. Astro. Soc. 475, 1427-1446 (2018).

26. Law, C. J. et al. A Multi-telescope Campaign on FRB 121102: Implications for the FRB Population. Astrophys. J. 850, 76 (2017).

27. Michilli, D. et al. An extreme magneto-ionic environment associated with the fast radio burst source FRB 121102. Nature 553, 182-185 (2018).

28. Keane, E. F. et al. The host galaxy of a fast radio burst. Nature 530, 453-456 (2016).

29. Scholz, P. et al. The Repeating Fast Radio Burst FRB 121102: Multi-wavelength Observations and Additional Bursts. Astrophys. J. 833, 177 (2016).

30. Macquart, J. P. et al. Fast Transients at Cosmological Distances with the SKA. Advancing Astrophysics with the Square Kilometre Array (AASKA14) 55 (2015).

Data availability Raw data files, (totalling 1 PB) are archived on tape at the Pawsey Centre. Cut-outs of the raw data, in pulsar filterbank format (http://sigproc.sourceforge.net) are available on the CSIRO 
data access portal through https://doi.org/10.25919/5b6ae6b515850. Other data products are available on request from R.M.S. (rshannon@swin.edu.au).

Code availability The code used to conduct the FRB searches, FREDDA, will be publicly released shortly, but a pre-release version is available from K.W.B. (keith.bannister@csiro.au). Detections were processed using the dspsr (h/tp://dspsr.sourceforge.net) and psrchive (h/tp://psrchive.sourceforge.net) software packages for analysing pulsar and FRB data.

Supplementary Information Guide The supplementary information is a single document comprising 6 supplementary sections, 26 supplementary references, 19 supplementary tables, and 28 supplementary figures.

Acknowledgements We thank the ATNF engineering and technical staff for their help supporting these observations, and especially thank the staff of the Murchison Radio-astronomy observatory. We thank C. Flynn, P. Edwards, N. Tejos, and V. McIntyre for comments on the manuscript, and members of the CRAFT team for discussions. We thank the MWA principal engineer, R. Wayth, for access to the Galaxy supercomputer GPU cluster. R.M.S. and S.O. acknowledge Australian Research Council (ARC) grant FL150100148. R.M.S. also acknowledges support through ARC grant CE170100004. Parts of this research were conducted by the ARC Centre of Excellence for All Sky Astrophysics (CAASTRO, CE110001020). This research was also supported by the ARC through grant DP18010085. The Australian SKA Pathfinder and Parkes radio telescopes are part of the Australia Telescope National Facility which is managed by CSIRO. Operation of ASKAP is funded by the Australian Government with support from the National Collaborative Research Infrastructure Strategy. ASKAP uses the resources of the Pawsey Supercomputing Centre. Establishment of ASKAP, the Murchison Radio-astronomy Observatory and the Pawsey Supercomputing Centre are initiatives of the Australian Government, with support from the Government of Western Australia and the Science and Industry Endowment Fund. We 
acknowledge the Wajarri Yamatji people as the traditional owners of the Observatory site. This research has made use of the NASA/IPAC Extragalactic Database (NED) which is operated by the Jet Propulsion Laboratory, California Institute of Technology, under contract with the National Aeronautics and Space Administration.

Author Contributions $\quad$ K.W.B. led the development the CRAFT data acquisition system. R.M.S., J.P.M, and K.B. designed the survey. R.M.S., J-.P.M, K.W.B. and R.D.E drafted the manuscript. R.M.S. and K.W.B. conducted the observations with assistance from A.H. and M.V. K.W.B. designed the search code. K.W.B., C.W.J., S.O, H.Q., and M.S. verified survey efficiency. R.M.S., with discussions with J.R.A., implemented the FRB localisation algorithm. R.M.S., J.-P.M., and R.D.E interpreted the fluence and dispersion measure distributions of the population. C.W.J., S.O., and J.-P.M. interpreted the nonrepetition of the ASKAP sample and compared it to the repeating FRB. R.M.S. and S.O. led searches for follow-up bursts at Parkes. E.M.S. studied the optical fields surrounding the detected FRBs. R.J.B., M.B., A.J.B., J.D.B., A.P.C., C.H., A.H., M.L., M.M,D.M., M.A.P, E.R.T., J.T., M.V., and M.T.W. contributed to the development and commissioning of the CRAFT observing mode. J.R.A., C.S.A., M.E.B., J.D.C., G.G., G.H., and C.J.R. contributed to ASKAP commissioning and early science.

Competing Interests The authors declare that they have no competing financial interests.

Correspondence Correspondence and requests for materials should be addressed to R.M.S. (email: rshannon@swin.edu.au) or J.-P.M. (email: J.Macquart@ curtin.edu.au) 


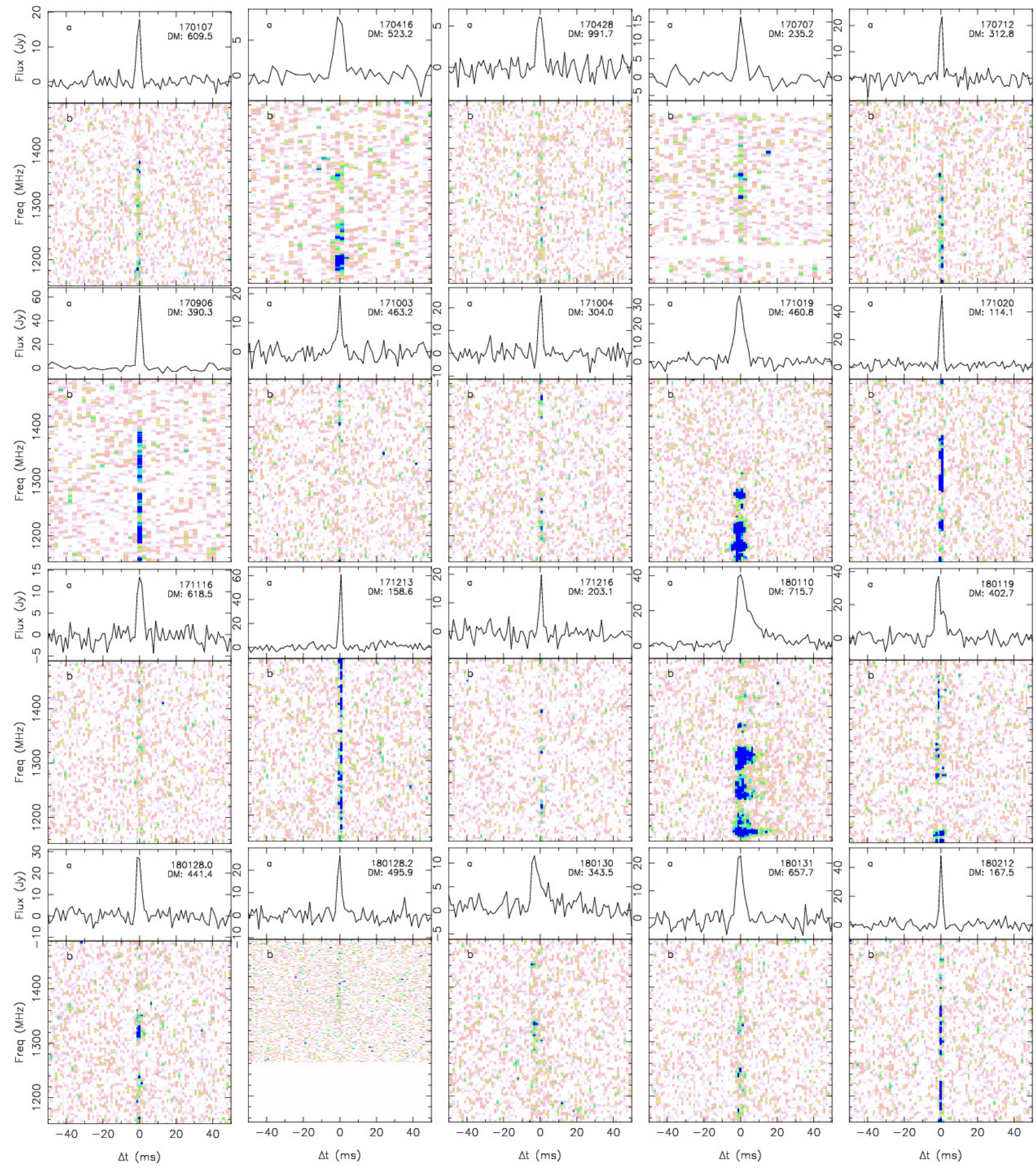

Figure 1: Pulse profiles and dynamic spectra of ASKAP fast radio bursts. The FRB names and dispersion measures (DM) in units of $\mathrm{pc} \mathrm{cm}^{-3}$ are listed in the panels showing the pulse profiles (Panels a). The spectra (Panels b) have been dedispersed to the maximum-likelihood dispersion measure. The colour scale is set to range from the mean to $4 \sigma$ of the off-pulse intensity. 


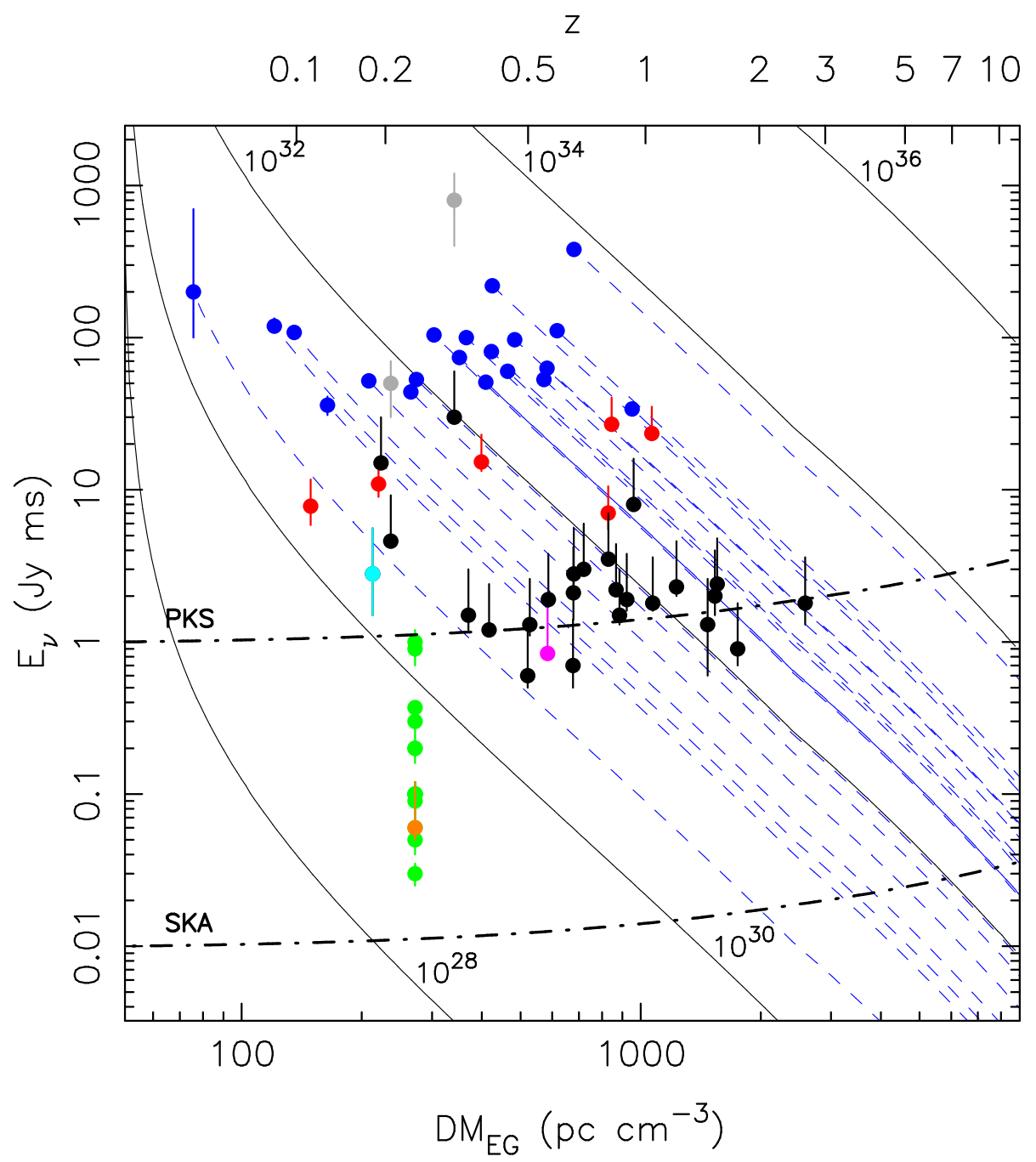

Figure 2: The fast radio burst fluence-extragalactic dispersion measure distribution. The extragalactic dispersion measure, $\mathrm{DM}_{\mathrm{EG}}$, is corrected for the inferred Milky-Way contribution. The coloured circles show FRBs detected with ASKAP (blue), Parkes (black), UTMOST (red), Green Bank Telescope (magenta), and Arecibo radio telescopes (orange). We also highlight the Parkes FRB candidate 010621 (cyan) which may be a Galactic source. Beam-corrected fluences have been estimated for two Parkes FRB ${ }^{18}(150807,010724)$ and are plotted in gray. Repeated pulses ${ }^{29}$ from FRB 121102 are displayed in green. Uncertainties in beam fluence differ by telescope. For ASKAP and burst from the repeating FRB 121102, we show $1 \sigma$ (67\% confidence) upper and lower limits. For other non-repeating FRBs lower limits are $1 \sigma$, but upper limits are twice detected fluence to reflect uncertainty in burst position within antenna pattern. The upper horizontal axis shows the redshift assuming a homogenously distributed intergalactic plasma $\frac{10}{10}$, and a host contribution of $50(1+z)^{-1} \mathrm{pc} \mathrm{cm}^{-3}$, as discussed in the text. The blue dashed curves show the expected fluences for the ASKAP-detected bursts if they were detected at larger distances (see supplementary section 4). The 1 black curves show contours of constant spectral energy density, in units of erg $\mathrm{Hz}^{-1}$. The dash-dotted lines are lines of constant fluence, after accounting for redshift dependent time dilation, denoting $10 \sigma$ sensitivities of the Parkes radio telescope and the mid-frequency first-phase component of the future Square Kilometre Arra ${ }^{30}$ to $1 \mathrm{~ms}$ bursts. Further details of the data used can be found in 


\begin{tabular}{|c|c|c|c|c|c|c|c|c|c|c|c|}
\hline FRB & $\begin{array}{c}\text { Time } \\
\left(\mathrm{TAI}^{(1)}\right)\end{array}$ & $\begin{array}{c}\mathrm{DM} \\
\left(\mathrm{pc} \mathrm{cm}^{-3}\right)\end{array}$ & $\begin{array}{c}E_{\nu} \\
(\mathrm{Jy} \mathrm{ms})\end{array}$ & $\begin{array}{c}\text { R.A. } \\
(\text { hh:mm })^{(2)}\end{array}$ & $\begin{array}{c}\text { Dec. } \\
(\mathrm{dd}: \mathrm{mm})^{(2)}\end{array}$ & $\begin{array}{c}g_{l} \\
\text { (deg.) }\end{array}$ & $\begin{array}{c}g_{b} \\
\text { (deg.) }\end{array}$ & $\begin{array}{c}w \\
(\mathrm{~ms})\end{array}$ & $\mathrm{S} / \mathrm{N}^{(3)}$ & $\begin{array}{l}T_{\text {obs }} \\
\text { (d) }\end{array}$ & $\begin{array}{l}T_{ \pm 15} \\
(\mathrm{hr})\end{array}$ \\
\hline 170107 & 20:05:45.1393(1) & $609.5(5)$ & $58(3)$ & $11: 23.3(7)$ & $-05: 00(10)$ & 266.0 & 54.1 & $2.4(2)$ & 16.0 & 27.9 & 15 \\
\hline 170416 & 23:11:49.7994(2) & $523.2(2)$ & $97(2)$ & $22: 13(1)$ & $-55: 02(9)$ & 337.6 & -50.0 & $5.0(6)$ & 13.0 & 16.2 & 31 \\
\hline 170428 & 18:03:11.7003(2) & $991.7(9)$ & $34(2)$ & $21: 47(2)$ & $-41: 51(20)$ & 359.2 & -49.9 & $4.4(5)$ & 10.5 & 31.6 & 22 \\
\hline 170707 & $06: 18: 11.3548(2)$ & $235.2(6)$ & $52(3)$ & $02: 59(2)$ & $-57: 16(20)$ & 269.1 & -50.5 & $3.5(5)$ & 9.5 & 11.3 & 56 \\
\hline 170712 & $13: 22: 54.39488(8)$ & $312.79(7)$ & $53(2)$ & $22: 36(1)$ & $-60: 57(10)$ & 329.3 & -51.6 & $1.4(3)$ & 12.7 & 7.7 & 32 \\
\hline 170906 & 13:07:33.48832(8) & $390.3(4)$ & 74(7) & 21:59.8(4) & $-19: 57(10)$ & 34.2 & -49.5 & $2.5(3)$ & 17.0 & 32.7 & 95 \\
\hline 171003 & 04:08:00.78117(9) & $463.2(1.2)$ & $81(5)$ & $12: 29.5(7)$ & $-14: 07(20)$ & 283.4 & 46.3 & $2.0(2)$ & 13.8 & 27.3 & 67 \\
\hline 171004 & 03:24:16.2501(1) & $304.0(3)$ & $44(2)$ & $11: 57.6(8)$ & $-11: 54(10)$ & 282.2 & 48.9 & $2.0(3)$ & 10.9 & 30.5 & 84 \\
\hline 171019 & $13: 27: 17.09738(1)$ & $460.8(1.1)$ & $219(5)$ & $22: 17.5(5)$ & $-08: 40(7)$ & 52.5 & -49.3 & $5.4(3)$ & 23.4 & 17.6 & 57 \\
\hline 171020 & $10: 28: 35.59870(4)$ & $114.1(0.2)$ & $200_{-100}^{+500}$ & $22: 15(3)$ & $-19: 40(40)$ & 29.3 & -51.3 & $1.7(2)$ & 19.5 & 32.7 & 95 \\
\hline 171116 & 15:00:10.3052(2) & $618.5(5)$ & $63(2)$ & $03: 31.0(6)$ & $-17: 14(10)$ & 205.0 & -49.8 & $3.2(5)$ & 11.8 & 45.7 & 102 \\
\hline 171213 & $14: 23: 17.46705(3)$ & $158.6(2)$ & $118(15)$ & $03: 39(2)$ & $-10: 56(20)$ & 200.6 & -48.3 & $1.5(2)$ & 25.1 & 35.5 & 118 \\
\hline 171216 & $17: 59: 47.82229(9)$ & $203.1(5)$ & $36(6)$ & $03: 28(1)$ & $-57: 04(10)$ & 273.9 & -48.4 & $1.9(3)$ & 8.0 & 7.7 & 26 \\
\hline
\end{tabular}




\begin{tabular}{|c|c|c|c|c|c|c|c|c|c|c|c|}
\hline $180110^{(4)}$ & 07:35:11.9590(1) & $715.7(2)$ & $380(10)$ & $21: 53.0(7)$ & $-35: 27(7)$ & 7.8 & -51.9 & $3.2(2)$ & 35.6 & 37.7 & 154 \\
\hline 180119 & $12: 25: 07.7476(2)$ & $402.7(7)$ & $100(3)$ & $03: 29.3(5)$ & $-12: 44(8)$ & 199.5 & -50.4 & $2.7(5)$ & 15.9 & 35.5 & 125 \\
\hline 180120.0 & 01:00:15.6179(1) & $441.4(2)$ & $51(2)$ & $13: 56(1)$ & $-06: 43(20)$ & 326.7 & 52.2 & $2.9(3)$ & 12.4 & 21.6 & 102 \\
\hline 180120.2 & 04:54:03.7962(1) & $495.9(7)$ & $60(2)$ & $22: 22(2)$ & $-60: 15(11)$ & 327.8 & -48.6 & $2.3(2)$ & 9.6 & 11.3 & 62 \\
\hline 180130 & 04:56:06.9932(1) & $343.5(4)$ & $104(2)$ & 21:52.2(9) & $-38: 34(10)$ & 5.9 & -51.8 & $4.1(1.0)$ & 10.3 & 37.7 & 120 \\
\hline 180131 & $05: 45: 42.3207(2)$ & $657.7(5)$ & $114(4)$ & 21:49.9(8) & $-40: 41(8)$ & 0.6 & -50.7 & $4.5(4)$ & 13.8 & 31.6 & 103 \\
\hline 180212 & 23:45:41.39991(9) & $167.5(5)$ & $108(10)$ & $14: 21(2)$ & $-03: 35(30)$ & 338.3 & 50.0 & $1.81(6)$ & 18.3 & 21.9 & 79 \\
\hline
\end{tabular}

Table 1:

Table 1: Properties of ASKAP FRBs. Uncertainties are listed in parentheses. $g_{l}$ : Galactic longitude of beam centre. $g_{b}$ : Galactic latitude of beam centre. $w$ : burst width. $T_{\text {obs }}$ : total observation time on field. $T_{ \pm 15}$ : Time on field within $\pm 15 \mathrm{~d}$ of FRB. (1) Burst arrival time referenced to a frequency of $1297.5 \mathrm{MHz}$ (2) Position uncertainties are $90 \%$ confidence limits and referred to the epoch J2000. Posterior localization regions are available as supplementary files. (3) S/N is the signal-to noise ratio reported in the primary beam by the search algorithm. (4) Quoted errors on fluence represented a 90\% confidence limit. (5) Pulse widths after deconvolution of exponential pulse broadening function. (See supplementary section 1). 


\section{Supplementary Information}

\section{Data set and data analysis:}

Searches were conducted as part of the Commensal Real-time ASKAP Fast Transient ${ }^{?}$ (CRAFT) early science observations (project code AS039). Our search strategy and data analysis largely follow the strategy as reported in the discovery of the first ASKAP-detected FRB ${ }^{7}$.

Searches have been conducted using a number of antennas in the array, with availability limited by commissioning activities and other early-science projects. From March-June 2017, observations were conducted in campaigns using the main array: antennas directly interfaced to the telescope correlator and used for commissioning interferometric and synthesis imaging modes for ASKAP. Up to 12 antennas were available for these searches. From June 2017 to present, observations have been conducted using the commissioning subarray, a set of antennas equipped with phased-array feed? (PAF) and digital subsystems suitable for recording ASKAPCRAFT fly's eye data?, but not from the correlator. Over the course of the survey, this sub-array increased in size from 5 to 9 available antennas as additional equipment was integrated.

From 2017 March our search strategy changed from a square beam configuration to a hexagonal close pack configuration (see Figure ??). In both cases the beam separation was $0.9 \mathrm{deg}$.

Over this time, nearly all of the searches were conducted in 57 fields at Galactic latitudes of $|b|=|50| \pm 5$ deg. Observations of these fields with 55 or 57 min duration were alternated with 3 or 5 -minute scans of known pulsars to check system performance and assess survey 
efficiency. Fields were assigned automatically by the telescope scheduling software. The selection algorithm was modified on 15 July 2017, to select fields that were close together and at high elevation angle. This was chosen to maximize the sensitivity of a low radiofrequency shadowing campaign with the Murchison Wide Field Array telescope, the results of which will be reported elsewhere. As a consequence of the prioritisation in the selection algorithm, there is large variation in the on-sky time in different fields, with the number of visits for each field ranging from 17 to 1309 , with a median of 570 .

Searches were conducted with a central frequency of $1320 \mathrm{MHz}$. The CRAFT data streams are dynamic spectra, produced in the digital beamforming subsystem, providing a total bandwidth of $336 \mathrm{MHz}$, with $1 \mathrm{MHz}$ spectral resolution and $1.26 \mathrm{~ms}$ temporal resolution in pseudo total intensity (i.e., the sum of the $X X$ and $Y Y$ linearly polarized intensities). These were summed together without any calibration. While beam centre gains between the two polarizations vary by less $\operatorname{than}^{6} 10 \%$, the beam shapes between the two can vary significantly; calibrating this is an active field of research within the ASKAP commissioning team. Data were recorded using methods nearly identical to those reported previously ${ }^{7}$. Data taking machines were upgraded twice during the course of the searches. Improvements were made to hardware tuning to mitigate network-related failures to record data.

The dynamic spectra were analysed identically to methods presented previously, using the graphical-processor-unit-accelerated search algorithm refered to as $F R E D D A^{7}$. Improvements were made to the code to better mitigate interference through the subtraction of a nondispersed time series and flag satellite interference. Satellite interference from GPS satellites transmitting in the band is the only significant form of interference persistent throughout the searches. 
This could be identified as it is confined to a band of frequencies (dependent on to which GPS service the satellite belonged). After flagging for this interference, two impulsive inteferers remained that affected a small fraction (1\%) of the data. One was impulsed chirped interference is occasionally produced by diagnostic monitoring of the PAF backplane. It produced candidate bursts with typically high DM ( $\left.>3000 \mathrm{pc} \mathrm{cm}^{-3}\right)$ at the polling interval of $35 \mathrm{~s}$ and was eliminated by disabling the monitoring during search observations. Occasional electrical storms would also cause candidates to be detected and would also be identified as episodes of excess low dispersion measure candidates, and could be correlated with on-site weather monitoring systems.

The searches were mostly conducted with on site computing facilities with a typical latency of $<24 \mathrm{hr}$ between observation and discovery; a small fraction of the data were searched on a GPU cluster at the Pawsey Centre (http: //pawsey.org.au), in Perth, with higher latency.

The total exposure is $5.1 \times 10^{5} \mathrm{deg}^{2} \mathrm{~h}$. This is the product of the total survey time, effective field of view, and the estimated survey efficiency. The total survey time (counting each antenna individually) was $32,840 \mathrm{hr}$. To calculate the exposure time we assume an effective field of view of $20 \mathrm{deg}^{2}$, which is the nominal equal power field of view ${ }^{?}$. This is smaller than the nominal field of view $30 \mathrm{deg}^{2}$, but a more appropriate value for comparison of survey rates because it accounts for the variations in sensitivity across the primary PAF beams. We assume that the observing efficiency is $80 \%$, which is the efficiency of detecting pulses from the pulsars in the $3-5$ minute hourly scans. 
We find no evidence for instrumental or terrestrial origin of the bursts. Bursts were detected in 9 unique antennas, and 19 unique beams, and across a wide range of time-of-day, antenna azimuth and elevation angles, solar angles and seasons. While some $30 \mathrm{deg}^{2}$-fields had multiple detections, they typically had larger-than-average observing times on them (a consequence to the uneven sampling of the fields, as mentioned above). Importantly the bursts show multiple beam detections, and the detections are consistent with arising from the antenna far field. Local interference would be present in more, if not all, of the PAF beams. The brightest burst, FRB180110, shows evidence for an exponential scattering tail, with the tail width varying with frequency as would be expected for propagation through a turbulent plasma. The strongest evidence for the bursts being not produced by interference will be through interferometric localization, a mode which is currently being commissioned.

The candidates are also inconsistent with being produced by thermal noise. A large number of trials have been searched, which we approximate to be $N_{\text {trial }} \approx T_{\text {obs }} R_{\text {samp }} N_{\mathrm{b}} N_{\mathrm{dm}} N_{\mathrm{box}} \approx$ $1326 \times 7.1 \times 10^{6} \times 35 \times 4096 \times 32 \approx 5 \times 10^{17}$, where $T_{\text {obs }}$ is the number of antenna days of surveying, $R_{\text {samp }}$ is the number of samples per day, $N_{b}=35$ is the number of useable beams, $N_{\mathrm{dm}}=4096$ is the number of trial dispersion measures, and $N_{\text {box }}=32$ is the number of boxcar widths searched. The CRAFT data stream from beam 36 is corrupted and does not produce useful data.

However, the probability of a single $9.5 \sigma$ candidate arising from thermal noise alone is $P \approx 10^{-21}$. The expected rate of such candidates across the survey is therefore $\approx 5 \times 10^{-4}$. This is likely an overestimate of the probability as the expression for $N_{\text {trial }}$ assumes all the trials are independent, which is not the case for DM, width, and time trials. 
In addition to strong primary beam detections ( $\mathrm{S} / \mathrm{N}>9.5$, with the exception of FRB171216, as discussed below). We have chosen to report a $\mathrm{S} / \mathrm{N}$ of 9.5 as our completeness limit. At this level we were able to verify the FRB nature of the emission. In particular we are able to detect the dispersion of the pulses across the band. Furthermore, we are able to have confidence in the detections as at this level, most of the bursts were detected in multiple beams. 14 of 20 were detected strongly $(\mathrm{S} / \mathrm{N}>5)$ in other beams, while 19 out of 20 detected at $(\mathrm{S} / \mathrm{N}>3)$ in adjacent beams.

Below this level, it is still possible to be convinced of the FRB nature of the signals if they have moderate detections in two beams, such as FRB 171216, for which the $\mathrm{S} / \mathrm{N}$ was greater than 10 when two beams were added together. However we cannot claim that this event is from a complete portion of phase space because our false positive rate increases, so this has not yet been applied to the data set.

The search algorithms have been tested using both simulated data sets and through observations of radio pulsars. We created synthetic filterbank data sets with integration time and bandwidths that were identical to the ASKAP digital system. We generated dispersed pulses over a range of DMs from 0 to $3000 \mathrm{pc} \mathrm{cm}^{-3}$ in steps of $25 \mathrm{pc} \mathrm{cm}^{-3}$ with an intrinsic width of a single sample. To simulate pulses of differing width, we convolved the pulse in each channel with a Gaussian function of 1 sigma width of 2,3 , and $4 \mathrm{~ms}$, matching the widths of the pulses detected at ASKAP and the majority of those detected at Parkes. We added Gaussian noise at level which yields a $\mathrm{S} / \mathrm{N}$ of each pulse of 9.5 , assuming a matched filter response to the whole pulse. Each dataset of a given DM and width comprised 100 pulses. We searched these data with FREDDA with a S/N threshold of 7 . For widths of $\geq 2 \mathrm{~ms}$, we recovered the pulses with a 
detection rate of $>90 \%$, and false positive $<1 \%$ for all DMs. For narrower pulses, we expect to lose $\mathrm{S} / \mathrm{N}$ and recovery due to dilution with the sample integration. Understanding the instrument response to narrow low-DM pulses (narrower than those typically detected at Parkes) would require simulation of voltages of dispersed pulses, channelised by polyphase filters, detected and integrated as in the CRAFT mode. The loss of sensitivity to low-DM narrow pulses is discussed further in supplementary section 5, in relation to differences in Parkes and ASKAP detection rates.

We searched these data with our search algorithm with a $\mathrm{S} / \mathrm{N}$ threshold of 7 sigma. For widths of $\geq 2 \mathrm{~ms}$, we recovered the pulses with a detection rate of $>90 \%$, and false positive $<1 \%$ for all DMs. For a width of $1 \mathrm{~ms}$ (less than a sampling time), the detection rate was $50 \%$ at $\mathrm{DM}=75 \mathrm{pc} \mathrm{cm}^{-3}$ and rose to $90 \%$ at $\mathrm{DM}=1000 \mathrm{pc} \mathrm{cm}^{-3}$. The loss in sensitivity for pulses narrower than the sampling time is an intrinsic property of any dedispersion search. The optimal delays applied to a given channel should differ depending on where the pulse begins during an integration. The algorithm computes channel delays optimised for a pulse beginning at the center of an integration, thus pulses beginning at the beginning or end of an integration are smeared over two integrations. This effect is has the most severe effect for narrow pulses at DMs where DM smearing is not large.

We also have tested the detection pipeline using pulsar data, which was also analysed by a computationally simpler algorithm using a matched filter against the known pulsar properties (pulse width, DM, and approximate Gaussian profile). For the pulses analysed, the pipeline detected the same pulses at $>90 \%$ the $\mathrm{S} / \mathrm{N}$ reported by the matched filter, at $100 \%$ efficiency for all pulses above threshold. 
We tested the recovery rate in survey data through hourly observations of the bright pulsars J1644-4559 and J0835-4510. These observations were of 3 or 5 min duration and were processed identically to our high galactic latitude fields. We find that we recover $80 \%$ of the pulses, with the remaining $20 \%$ lost due to satellite interference. We account for the $20 \%$ loss in estimating our total survey time. After automatic flagging of the data for satellite interference, and removing a few observations (totally less than $1 \%$ of the survey) affected by the impulsive interference listed above, manual inspection of all the high latitude candidates was conducted, with fewer than 10 candidates produced per day of observation.

Supplementary tables ?? to ?? show the signal-to-noise (S/N) ratios in the primary beam and adjacent beams for FRBs 170416 to 180212. The observations of FRB 170107 were reported previously ${ }^{7}$. We calculate the $\mathrm{S} / \mathrm{N}$ using the $N$ bins in the frequency-averaged pulse profile, an estimate of $\sigma$ using off-pulse baseline. It this case

$$
S / N=\frac{\sum F_{i}}{\sigma \sqrt{N}}
$$

Secondary detections and upper limits enabled FRBs to be localized to $\approx 10 \times 10$ arcmin regions, much smaller than the $1.1 \mathrm{deg}$ antenna (primary) beam width for ASKAP at these frequencies. The posterior localization regions are displayed in greyscale relative to the beam positions in Figures ?? to ??, with the intensity of the greyscale proportional to posterior probability; these regions are available in absolute sky coordinates as supplementary files in FITS format (https://fits.gsfc.nasa.gov/).

A physical flux scale was applied to data sets by assuming the noise in our data sets is dominated by thermal noise, and the system equivalent flux density $S_{\mathrm{sys}}$ is $2000 \mathrm{Jy}$, consistent 
with interferometric measurements of feed sensitivity ${ }^{6}$ and calibration against simultaneous observations with the Parkes telescope made of PSR B1641-45. In this case the root-meansquare noise is $S_{\mathrm{sys}} / \sqrt{2 \Delta \nu \Delta t}$, where $\Delta \nu$ is the spectral bandwidth and $\Delta t$ is the time resolution. We corrected the integrated pulse profiles (panels labelled $a$ in Fig. 1) for off axis attenuation assuming a fixed frequency of $1.3 \mathrm{GHz}$ and the mean offset position of the burst from its primary-beam detection. We did not correct the fluence estimates for a $10 \%$ increase in $S_{\text {sys }}$ above $1.4 \mathrm{GHz}^{?}$. This would result in a small correction as most of the band we observe in is below this frequency, and is smaller than the systematic uncertainties by at least a factor of 5. We have however corrected for this effect in the pulse profiles plotted in Figure 1 and in the spectral index estimates.

Burst widths were measured using the frequency integrated spectrum (see Table ??) A non-linear Levenberg-Macquardt fitting algorithm, as implemented in the gnuplot package, was used to measure pulse widths. Most bursts were adequately fitted using a Gaussian function, given the low signal-to-noise ratio of our data. However two bursts (FRBs 180110 and 180130) show evidence for scattering. In this case, the functional form for the function was a Gaussian convolved with an exponential.

Details about specific discoveries are listed below:

FRB 170707: Digital beam weights were not successfully made for some channels, resulting in no signal being recorded for some channels in the burst.

FRB 170906: The data were affected by digitization overflow in the brightest portion of the pulse spectrum. The signal could be recovered by using a beam model and an adjacent beam 
detection, where the signal was weaker and therefore not affected by the overflow problem. This was fixed for subsequently detected FRBs. The correction relies on a good understanding of the ASKAP primary beam models distant from the source, so there are larger uncertainties in the peak brightness of the spectrum than for other bursts. One channel of the spectrum in Figure ?? near the peak of bright band drops suddenly; it is possible that this drop is associated with an uncorrected digital artefact.

FRB 171020: The dispersion measure of the burst $\left(\mathrm{DM}=114.1 \pm 0.2 \mathrm{pc} \mathrm{cm}^{-3}, 1 \sigma\right)$ is the lowest detected for an FRB in our sample and lowest for any published burst. The FRB was detected in a corner beam (see supplementary Figure ??); consequently the localization is poor. However, because the extra-Galactic DM is small, the burst likely arises from a nearby galaxy. In addition to the two galaxies listed in the main text, the galaxy cluster Abell 3852 also lies within the FRB uncertainty region. Although there is no published redshift for this cluster, we can estimate its redshift as $z \sim 0.21$ based on the value of $\mathrm{m} 10=19.1$ mag listed by ${ }^{?}$ and the empirical relationship between m10 and redshift derived by these authors. The galaxies in Abell 3852 are therefore too distant to be plausible hosts for this low-DM FRB.

FRB 171216: The burst was first identified as being an unusually temporally narrow candidate (only one sample in width), but has a $S / N=8.0$ below the completeness signalto-noise ratio limit of the survey $(S / N=9.5)$ for a single-beam search. It is therefore not included in tests of the cosmological nature of the distribution. Analysis of adjacent beams show that the burst has nearly equal signal-to-noise ratios in two adjacent beams, as listed in Table ??. The co-added signal to noise ratio from these two detections is 10.3 . This co-added pulse is displayed in Figs. 1 and ??. 
FRB 180110: The frequency-averaged spectrum of the burst has strong exponential morphology, which would be expected from a pulse scattered off of inhomegeneities in a turbulent plasma. The strength of the scattering (and hence the size of the tail) is highly chromatic. We subdivided the pulse into 8 channels and fitted each independently to a Gaussian convolved pulse of unknown amplitude and width, arrival time, and scattering time. The scattering time changes strongly with frequency (see supplementary Figure ??). We then fitted the scattering times as a function of frequency $\tau(\nu)=A\left(\nu / \nu_{0}\right)^{\beta}$, and found that $\beta=-3.5 \pm 0.6$ and $A=3.6 \pm 0.4 \mathrm{~ms}$ at a reference frequency of $\nu_{0}=1.4 \mathrm{GHz}$. Our results are consistent with the theoretically predicted values of $\beta=4.0-4.4$ expected for scattering in Kolmogorov media ${ }^{19}$ and also observed scattering indices which average to $3.9 \pm 0.2$ for pulsars in the Milky Way?

FRB 180119: This burst shows marginal evidence for a scatter broadened tail. The burst is too weak to search for frequency dependent variations as for FRB 180110. The broadening is estimated to be $2.8 \pm 0.5 \mathrm{~ms}$.

FRB 180128.2: The data were affected by strong interference in the lower portion of the band. These RFI-affected channels have been flagged in subsequent analyses.

FRB 180130: This burst shows marginal evidence for scatter broadened tail. The burst is too weak to search for frequency dependent variations as for FRB 180110. The broadening is estimated to be $6 \pm 1 \mathrm{~ms}$. 


\section{Analysis of the global spectral properties of the bursts:}

We formed spectra for each FRB by integrating the time samples over which the frequency averaged pulse shows detectable signal (nominally at the $1 \sigma$ noise level). We quote the amplitude of the spectra in fluence, and convert to physical units using an assumed value for the system equivalent flux density ( $S_{\mathrm{sys}}=2000 \mathrm{Jy}$, as discussed above $)$ and the measured level of off-pulse noise in the time series using standard methods. The spectra of the bursts can be found in Figure ?? (black lines). The red lines in the same figure show a smoothed version of the spectra. The gray lines show baseline off-pulse spectra taken within $100 \mathrm{~ms}$ of each FRB, which provide a guide for the noise level in the FRB spectra and show that data are in general not suffering from excess radio-frequency interference.

Before averaging, we corrected for chromaticity expected due to the beam pattern. This correction is small because the beam pattern densely samples the focal plane, with the beams overlapping well within the half power beam point. We also corrected the spectra for a $10 \%$ increase in $S_{\text {sys }}$ above $1.4 \mathrm{GHz}$.

We calculated the average spectra two different ways. In the first, we used natural weighting, in which we directly summed the frequency-dependent fluences for each burst. The average spectra for this can be seen in Figure ??. We fitted a power-law model to the spectra $\left(E(\nu) \propto \nu^{\beta}\right)$ using a nonlinear fitting algorithm, and found $\beta=-3.3 \pm 0.3$. We used the bootstrap technique? ${ }^{?}$ to assess how sample variation affects our estimate of the average spectral index. To do this, we formed average spectra, sampling with replacement from the 20 spectra. This formed 1000 composite spectra, each of which was fitted the same power law form for the 
flux density. We found that the median spectral index was $\beta=-3.33_{-0.31}^{+0.30}$, matching the fitted value.

In the second method, we weighted the bursts by the inverse of their signal to noise ratio to reduce the bias introduced in the spectra by the bright bursts. When we do this, we find $\beta=-1.8 \pm 0.3$.

In a third method, we formed the spectrum from the median of all 20 FRBs. In this case, we also find $\beta=-1.8 \pm 0.3$.

It is unclear what produces the steep average spectrum. It could be intrinsic to the emission mechanism. Alternatively it could be a secondary propagation effect. In this case, models such as lensing ${ }^{15}$ would need to predict a change in spectral occupancy across the band. Diffractive scintillation does not induce steep spectra. Our observations have a relatively small fractional bandwidth of $\approx 10 \%$. Broader band observations would strengthen the case for a steep spectral index. Similarly the study of the FRB population at lower frequencies (for example by observing with ASKAP in the lower band or with the other facilities such as the Canadian HI intensity mapping experiment) would also be able to confirm the population has on average a steep spectral index.

\section{Source-count analysis:}

To analyse the distribution of the bursts, we consider the 19 events above our completeness limit of a signal to noise ratio of 9.5. To examine the source distribution, we use the test statistic? $\sqrt[22]{22}$ $V / V_{\max }$, which is the average fractional volume within which the sources reside relative to 
the volume within which they could have been detected. The statistic is calculated from the measured signal-to-noise ratios $\left((S / N)_{i}\right)$ :

$$
\frac{V}{V_{\max }}=\frac{1}{N_{\mathrm{FRB}}} \sum_{i}^{N_{\mathrm{FRB}}}\left(\frac{(S / N)_{i}}{(S / N)_{\min }}\right)^{-3 / 2} .
$$

For a population of sources uniformly distributed throughout Euclidean (flat) space-time, the ensemble average value is $\left\langle V / V_{\max }\right\rangle=0.5$. One advantage of using $V / V_{\max }$ as a test statistic is that it is agnostic to burst width, which varies across the population due to physical and instrumental effects. If there is a correlation between burst width and fluence there could be secondary biases introduced in $V / V_{\max }$ because narrow (but temporally resolved) pulses are easier detect than broader pulses. This will be yet more important when comparing burst populations detected by different telescopes or observing systems. A fuller discussion of the utility of the V/Vmax test in the context of FRBs was previously presented ${ }^{22}$.

The statistic is also independent of the intrinsic luminosity function of the emitting objects, so is valid for both source classes that are standard candles, as well as those that have a broad luminosity function.

For our sample, we find $V / V_{\max }=0.58$. We calculated the probability that this distribution arises from Euclidean population through simulation. The detected population will depend primarily on the intrinsic burst fluence $E(\nu)$ but secondarily on the width $w$, and dispersion measure DM. As the purposes of the simulations are to examine a population of local bursts, it is sufficient to assume that these quantities are independent. We assume the population of bursts with fluences that follow a power-law distribution $P(>E(\nu)) \propto E(\nu)^{\alpha}$. We will assume that the pulses are Gaussian in shape and that the intrinsic width $w_{i}$ is Normally distributed about some mean $\mu_{w}$ with variance $\sigma_{w}^{2}$. We will assume that the dispersion mea- 
sures are also Gaussian distributed with a mean value $\mu_{\mathrm{DM}}$ and variance $\sigma_{\mathrm{DM}}^{2}$. This fit well models the current ASKAP FRB dispersion-measure distribution. We note that our simulations confirm known properties of the $V / V_{\max }$ statistic. For example, the standard deviation of the statistic is $\approx 1 / \sqrt{12 N}$, where $N$ is the size of the population used to test the statistic.

The observed pulse width is instrumentally broadened if the dispersion smearing across a single channel is large or the pulse is narrower than the time sampling $t_{\mathrm{samp}}$. The functional form of this broadening will depend on the impulse response of the spectrometer. We will assume that the effects can be treated as a convolution, and that the impulse response function for both effects are Gaussian as well. In this case the pulse width is

$$
w_{\mathrm{obs}}=\sqrt{w_{\mathrm{int}}^{2}+w_{\mathrm{DM}}^{2}+t_{\mathrm{samp}}^{2}}
$$

where the dispersion smearing is $w_{\mathrm{dm}} 8.15^{-6} \mu \mathrm{s} \mathrm{DM} \Delta \nu \nu^{-3}$, where $\mathrm{DM}$ is expressed in $\mathrm{pc} \mathrm{cm}^{-3}$, channel bandwidth $\Delta \nu$ is expressed in $\mathrm{MHz}$ and observing frequency in GHz.

To model the spectral modulation we multiplied the fluence by a random gain factor $g$ that was exponentially distributed ( $\chi^{2}$ with two degrees of freedom), consistent with diffractive scintillation at high Galactic latitude, where scintillation bandwidths are comparable to the total observing bandwidth ?

The observed flux density in time $w_{\text {obs }}$ is $g E / w_{\text {obs }}$ Therefore the signal-to-noise ratio is

$$
\mathrm{S} / \mathrm{N}=\left(\frac{g E}{w_{\mathrm{obs}}}\right)\left(\frac{\sqrt{2 B w_{\mathrm{obs}}}}{S_{\mathrm{sys}}}\right)
$$

where $B$ is the total observing bandwidth and the system equivalent flux density $S_{\text {sys }}$ factors in aperture efficiency. 
To test the consistency of the a Euclidean source-count distribution with the observations, we first generated a large number $\left(>10^{4}\right)$ bursts from that distribution that satisfied our detection S/N. From that sample, we selected (with replacement) 19 events. We then calculated the $V / V_{\max }$ statistic for the 19 events. We repeated this process 1000 times, to build up an expected distribution for the test statistic statistic. We then determined the fraction of times the test statistic exceeded the value of $V / V_{\max }=0.58$ we measured. We note that these simulations assume that the population is both local and homogeneous. For a very nearby population in which sources trace the local large scale structure - closer than a few hundred Mpc so as not to average over the $30 \mathrm{Mpc}$ typical void size - this need not be the case. If the repeating FRB and the non-repeating bursts are in the same population; the distance scale to the former also points to a more distant population. As discussed in the main paper there are lines of evidence suggesting the populations are different. Also as discussed below, the dispersion measure distribution differences between the Parkes and ASKAP suggests a distant population.

Over a wide range of values for the parameters describing the width $\left(\mu_{w}\right.$ and $\left.\sigma_{w}\right)$ and DM ( $\mu_{\mathrm{DM}}$ and $\sigma_{\mathrm{DM}}$ ), we found that for a Euclidean source count distribution, the $V / V_{\max }$ values were inconsistent with our measurement at the $\approx 90 \%$ confidence level. In particular for values that match the observed DM and (deconvolved) pulse width distributions of the FRBs in our sample $\left(\mu_{\mathrm{DM}}=421 \mathrm{pc} \mathrm{cm}^{-3}, \sigma_{\mathrm{DM}}=220 \mathrm{pc} \mathrm{cm}^{-3}, \mu_{w}=2.5 \mathrm{~ms}, \sigma_{w}=1.6 \mathrm{~ms}\right)$, we find the probability that the fluence distribution is inconsistent with Euclidean is 88\%, using a onesided test (i.e., $\left.P\left(V / V_{\max }>0.58\right)\right)$. A one sided test is appropriate as values of $V / V_{\max }<0.5$ are not expected. The distribution of simulated test statistic (black line) assuming a Euclidean energy distribution (black line) and its expected distribution based on the assumption that $\sigma=$ $1 / \sqrt{12 N}$ are displayed in supplementary Figure ??. In supplementary Figure ??, we show 
the results of simulations of pulses with ASKAP-like DM distributions derived from fluence distributions with $\alpha=-1.5$ (Euclidean) and $\alpha=-2.1$. The ASKAP fluences produce fewer bright FRBs than expected from a Euclidean distribution, and better match the steeper source counts. The top panels ( $a$ and $d$ ) show the DM distributions of the ASKAP (blue) and Parkesdetected FRBs (red), in addition to the simulated ASKAP population (blue). As can be seen the simulated and observed ASKAP distributions match well.

To test which values of source count slope $(\alpha)$ were consistent with the observed value of the test statistic, we simulated event lists at a range of source count distributions and recomputed the $V / V_{\max }$ distribution. We found the value of $\alpha$ for which $V / V_{\max }>0.5850 \%$, $17 \%$, and $83 \%$ of the time, and used these values to define the maximum likelihood and $67 \%$ confidence region for $\alpha$.

The value of the $V / V_{\max }$ test statistic does not change significantly when setting a higher threshold. When setting the threshold to be $S / N>15$, we find $V / V_{\max }=0.66 \pm 0.10$, consistent with that calculated at a $\mathrm{S} / \mathrm{N}$ of 9.5 .

\section{FRB fluence dispersion-measure distribution:}

In Figure 2, we have plotted the FRBs detected at Parkes?,?,?,?,?,?,?,1/2,5]16/17/25,28 at a frequency of $1.4 \mathrm{GHz}$; those detected at the upgraded Molonglo Telescope (UTMOST $, ?, ?, ?, 21$ ) at a central frequency of $0.843 \mathrm{GHz}$; and at the Green Bank telescope $\mathrm{e}^{200}$ at a frequency of $0.8 \mathrm{GHz}$. For the repeating FRB (121102), we have plotted both the detected fluence for FRB $121102^{?}$ at a frequency of $1.4 \mathrm{GHz}$, as well as the fluences of repeated bursts detected at observation 
frequencies close to $1.4 \mathrm{GHz}^{\frac{3}{29}}$. We have assumed published uncertainties are accurate for fluence lower limits. As there is large uncertainty in burst location within the beam pattern for non-ASKAP FRB discoveries, the upper limits are quoted at twice the reported fluence. We note that observations of the repeating FRB after it was discovered have more reliable measurements and in Figure 2, we show the reported error bars. For Parkes FRBs 150807 and 110724 we have highlighted fluences corrected by modelling multiple beam detections. FRB 110724 has the high but uncertain fluence $E_{\nu}=800 \pm 400 \mathrm{Jy} \mathrm{ms}$; uncertainties are dominated by instrumental effects.

The burst dispersion measures have been corrected for Galactic contributions using the NE2001 model $^{?}$ for the electron density of the Milky Way Disk. We also remove a nominal Milky Way halo contribution, a practice consistent with the DM calculation of the repeating $\mathrm{FRB}^{4}$. We empirically estimate a likely halo contribution of approximately $15 \mathrm{pc} \mathrm{cm}^{-3}$, based on the lowest dispersion measures of pulsars in the Large Magellanic Cloud pulsar survey ${ }^{?} ;$ the minimum DMs are 65, 68 and $69 \mathrm{pc} \mathrm{cm}^{-3}$ and NE2001 reports a Galactic contribution in this direction of $52 \mathrm{pc} \mathrm{cm}^{-3}$. For most halo models (e.g., based on X-ray absorption measurements ${ }^{\text {? }}$ ) the decline in the density beyond the $\sim 50 \mathrm{kpc}$ distance of the LMC provides at most a further 1-2 $\mathrm{pc} \mathrm{cm}^{-3}$ beyond this (the DM contribution at larger radii, $r$, is substantially smaller than the further contribution to the halo mass since each shell adds mass proportional to $r^{2}$ ). Other estimates of the halo contribution ${ }^{?}$ are within the $\sim 10 \mathrm{pc} \mathrm{cm}^{-3}$ uncertainty of the Milky Way DM contribution.

The conversion between dispersion measure and redshift has been calculated assuming concordance cosmology and a smooth distribution of electron density ${ }^{10}$, and assuming that the 
host dispersion measure contribution is $50(1+z)^{-1} \mathrm{pc} \mathrm{cm}^{-3}$. Bursts are likely to show large variations in host-galaxy DM contribution, with variations depending on the viewing geometry. At the mean viewing angle, host galaxy contribution to the DM has been estimated to be $30-40$ $\mathrm{pc} \mathrm{cm}^{-3}$ for elliptical and dwarf galaxies and $50-60 \mathrm{pc} \mathrm{cm}^{-3}$ for spiral galaxies ${ }^{9}$.

\section{Comparing the DM distributions from Parkes and ASKAP}

There is a notable difference in the DM distributions from Parkes and ASKAP. This difference is intrinsic to the FRB populations and not due to instrumental selection effects.

This can be demonstrated through the same simulation approach implemented for the source-count analysis. The modern Parkes system, which now dominates the Parkes detections has higher time $\left(\Delta t_{\mathrm{samp}}=64 \mu \mathrm{s}\right)$ and frequency resolution $(\Delta \nu=390 \mathrm{kHz})$ than the ASKAP system $\left(\Delta t_{\mathrm{samp}}=1.26 \mathrm{~ms}, \Delta \nu=1 \mathrm{MHz}\right)$. As a result, high dispersion measure pulses have larger instrumental broadening at ASKAP.

We use the same simulation procedures we used to examine the statistics of the $V / V_{\max }$ distribution to test the sensitivity of the system to pulses of differing widths and DM. We considered pulses with Euclidean source-count distribution. Instead of considering events with pulse widths and DM consistent with an ASKAP-like distribution, we use broader distributions to examine how selection bias could be affecting the ASKAP distributions. Therefore, we simulated pulses with uniform probability density of arising between 50 and $4000 \mathrm{pc} \mathrm{cm}^{-3}$, in $\log (\mathrm{DM})$. The purpose of this DM distribution is not to simulate a physical DM distribution but to allow plots of the detected DM distribution to be easily interpreted. Histograms of the 
distribution of the detected pulses in $\log (\mathrm{DM})$ directly show how sensitivity is lost with DM. Pulses were assumed to have widths between 0.1 and $10.0 \mathrm{~ms}$ with equal probability density in $\log ($ width)

While there is a difference in total rate detected by the two systems, there is no evidence for ASKAP losing a disproportionate number of high-DM events. Figure ?? shows the normalised dispersion measure distributions for events detected by ASKAP-like (blue) and Parkes-liked (red) backend systems. The figure shows that above a DM of $\approx 300 \mathrm{pc} \mathrm{cm}^{-3}$ the shapes are identical. This indicates that above this DM, both systems are losing events in equal proportion. Above the Parkes diagonal DM, the largest diagonal DM, both systems sensitivity decrease at the same rate with respect to DM. Therefore above this DM, any difference in the DM distribution reflects intrinsic properties of the bursts detected by the two telescopes. The only difference between the two systems is that the Parkes-like system would be expected to detect more low-DM events (a consequence of the higher time resolution of Parkes), which is also in complete disagreement with the observed DM distributions of the two telescopes.

\section{Interpreting the non-repetition of the ASKAP sample}

To limit the repetition properties of ASKAP FRBs, we assume that they all repeat with identical rates $R$ :

$$
R(S)=R_{0}\left(S / S_{0}\right)^{\gamma}
$$

Here, $R_{0}$ is the rate above the ASKAP detection threshold $S_{0}$ (nominally $46 \mathrm{Jy}$ assuming a width close to the median of $4 \mathrm{~ms}$ ). We note that the detection depends on pulse width. This scales to other fluence thresholds $S$ according to the cumulative repetition rate index $\gamma$. 
The probability of detecting no repeat pulses from any of the observed FRBs can be calculated as:

$$
p(\text { no repeats })=\prod_{i=1}^{N_{\mathrm{FRB}}}\left(1+R_{0} T_{i}\right) e^{-R_{0} T_{i} B_{i}^{\gamma}}
$$

where the terms in the product represent the Poisson probability of observing 0 or 1 bursts given a $T_{\mathrm{obs}}$ of $T_{i}$, and beam correction factor $B_{i}$. This allows ASKAP FRB repetition rates greater than once per 62 (35) [23] days to be excluded with 68\% (95\%) [99.7\%] confidence in a one-sided test for $\gamma=-1$. This value was chosen because it provides the steepest pulseenergy distribution consistent with the repeating FRBs, and thus provides the most conservative constraint on repetition within the ASKAP sample.

Equation ?? is used to scale to the sensitivity at which FRB 121102 has been observed, comparing to previous VLA observations ${ }^{26}$, for which a $7.4 \sigma$ threshold to each spectral window with $1 \sigma$ sensitivity of $5 \mathrm{mJy}$ over $5 \mathrm{~ms}$ produces $S_{\mathrm{VLA}}=184 \mathrm{mJy} \mathrm{ms}$. The resulting limits on $R(184 \mathrm{mJy} \mathrm{ms})$ are shown in Figure ??.

Law et al. ${ }^{26}$ report $\gamma_{\mathrm{VLA}}=-0.6_{-0.2}^{+0.3}$. Rates are taken from the late-2016 and early-2016 samples, reporting 9 (nominal uncertainty \pm 3 , i.e. $0.32 \pm 0.1 \mathrm{hr}^{-1}$ ) and 0 bursts, respectively. We exclude almost the entire late-2016 region in $R-\alpha$ space at $99.7 \%$ confidence, with a small region at $\gamma=-0.9$ being allowed. Performing a joint maximisation of $R$ to allow for fluctuations in both the observed VLA and ASKAP rates results in $R(184 \mathrm{mJy} \mathrm{ms})=0.18 \mathrm{hr}^{-1}$ for $\gamma=-0.9$, for which the probability product of ASKAP seeing no repeat bursts, and the VLA seeing at least nine, is $0.4 \%$. This demonstrates that ASKAP FRBs do not exhibit repetition with the same characteristics as FRB 121102. 
Comparisons between FRB 121102 and the limits on the repetition properties of ASKAP FRBs presented here should be taken with caution, due to the different observation bands, frequency structure, pulse duration, and dispersion measures. In particular, equation ?? would be more correctly formulated in terms of intrinsic source properties, so that non-observations of repeats from nearby (presumably low-DM) FRBs would be more significant. A more complete analysis would also include a model of the undetected repeating FRBs, since non-observations of multiple pulses from these will further limit the repetition rate through equation ??. Finally, note that any deviations from Poisson statistics in pulse arrival times will lead to clustering, and hence stronger limits on repetition for those FRBs which are necessarily detected in their active phase.

We note that our constraints on the repetition rate use a relatively high threshold for other bursts, comparable to the one used for blind FRB searches. Searches at lower thresholds are possible because they can focus on a narrow dispersion measure range in the pointings directed toward the FRBs. Sub-threshold searches are ongoing with effort being placed to characterize false alarms due to interference at these lower thresholds. 


\section{Supplementary References}

31. Macquart, J.-P. et al. The Commensal Real-Time ASKAP Fast-Transients (CRAFT) Survey. Publ.Astron.Soc.Australia 27, 272-282 (2010).

32. Bunton, J. D. \& Hay, S. G. Achievable field of view of chequerboard phased array feed. In Electromagnetics in Advanced Applications (ICEAA), 2010 International Conference on, 728-730 (IEEE, 2010).

33. Beresford, R. et al. Radio astronomy l-band phased array feed rfof implementation overview. In 2017 XXXIInd General Assembly and Scientific Symposium of the International Union of Radio Science (URSI GASS), 1-4 (2017).

34. Hay, S. G. \& O’Sullivan, J. D. Analysis of common-mode effects in a dual-polarized planar connected-array antenna. Radio Science 43 (2008). RS6S04.

35. Chippendale, A. P. et al. Recent developments in measuring signal and noise in phased array feeds at csiro. In 2016 10th European Conference on Antennas and Propagation (EuCAP), 1-5 (2016).

36. Abell, G. O., Corwin, H. G., Jr. \& Olowin, R. P. A catalog of rich clusters of galaxies. Astrophys. J. 70, 1-138 (1989).

37. Bhat, N. D. R., Cordes, J. M., Camilo, F., Nice, D. J. \& Lorimer, D. R. Multifrequency Observations of Radio Pulse Broadening and Constraints on Interstellar Electron Density Microstructure. Astrophys. J. 605, 759-783 (2004). 
38. Efron, B. Bootstrap methods: Another look at the jackknife. The Annals of Statistics 7, $1-26(1979)$.

39. Schmidt, M. Space Distribution and Luminosity Functions of Quasi-Stellar Radio Sources. Astrophys. J. 151, 393 (1968).

40. Rickett, B. J. Interstellar scintillation and pulsar intensity variations. Mon. Not. R. Astro. Soc. 150, 67 (1970).

41. Burke-Spolaor, S. \& Bannister, K. W. The Galactic Position Dependence of Fast Radio Bursts and the Discovery of FRB011025. Astrophys. J. 792, 19 (2014).

42. Thornton, D. et al. A Population of Fast Radio Bursts at Cosmological Distances. Science 341, 53-56 (2013).

43. Ravi, V., Shannon, R. M. \& Jameson, A. A Fast Radio Burst in the Direction of the Carina Dwarf Spheroidal Galaxy. Astrophys. J. 799, L5 (2015).

44. Petroff, E. et al. Identifying the source of perytons at the Parkes radio telescope. Mon. Not. R. Astro. Soc. 451, 3933-3940 (2015).

45. Shannon, R. M. et al. Real-time detection of a low-latitude Fast Radio Burst during observations of PSR J1545-4550. The Astronomer's Telegram 11046 (2017).

46. Price, D. et al. Detection of a new fast radio burst during Breakthrough Listen observations. The Astronomer's Telegram 11376 (2018).

47. Oslowski, S. et al. A second fast radio burst discovered with Parkes Telescope within 50 hours: FRB180311 in the direction of PSR J2129-5721. The Astronomer's Telegram 11396 (2018). 
48. Bailes, M. et al. The UTMOST: A Hybrid Digital Signal Processor Transforms the Molonglo Observatory Synthesis Telescope. Publ.Astron.Soc.Australia 34, e045 (2017).

49. Caleb, M. et al. The First interferometric detections of Fast Radio Bursts. Mon. Not. R. Astro. Soc. 458, 718-725 (2017).

50. Farah, W. et al. Detection of a highly scattered Fast Radio Burst at the Molonglo Radio Telescope. The Astronomer's Telegram 10867 (2017).

51. Farah, W. et al. Detection of a Fast Radio Burst at the Molonglo Radio Telescope. The Astronomer's Telegram 11675 (2018).

52. Spitler, L. G. et al. Fast Radio Burst Discovered in the Arecibo Pulsar ALFA Survey. Astrophys. J. 790, 101 (2014).

53. Cordes, J. M. \& Lazio, T. J. W. NE2001.I. A New Model for the Galactic Distribution of Free Electrons and its Fluctuations. ArXiv Astrophysics e-prints (2002).

54. Manchester, R. N., Hobbs, G. B., Teoh, A. \& Hobbs, M. The Australia Telescope National Facility Pulsar Catalogue. Astron. J. 129, 1993-2006 (2005).

55. Miller, M. J. \& Bregman, J. N. The Structure of the Milky Way's Hot Gas Halo. Astrophys. J. 770, 118 (2013).

56. Dolag, K., Gaensler, B. M., Beck, A. M. \& Beck, M. C. Constraints on the distribution and energetics of fast radio bursts using cosmological hydrodynamic simulations. Mon. Not. R. Astro. Soc. 451, 4277-4289 (2015). 


\section{Supplementary Tables}

Supplementary Table 1: FRB 170416 detections

\begin{tabular}{cccc} 
Beam & RA (J2000) & DEC (J2000) & S/N \\
& $(\mathrm{deg})$ & $(\mathrm{deg})$ & \\
\hline 15 & 332.24 & -54.834 & 7.8 \\
16 & 333.03 & -55.62 & 8.3 \\
20 & 333.01 & -54.06 & 2.0 \\
21 & 333.80 & -54.83 & 12 \\
27 & 334.54 & -54.04 & 2.0 \\
28 & 335.36 & -54.81 & 2.0 \\
\hline
\end{tabular}

Supplementary Table 2: FRB 170428 detections

Beam RA (J2000) DEC (J2000) S/N

\begin{tabular}{llll} 
& $(\mathrm{deg})$ & $(\mathrm{deg})$ \\
\hline 19 & 326.92 & -41.84 & 8.2 \\
20 & 327.53 & -42.62 & 0.9 \\
14 & 326.31 & -42.62 & 2.5 \\
13 & 325.71 & -41.83 & 1.4 \\
18 & 326.33 & -41.06 & 2.0 \\
25 & 327.52 & -41.06 & 1.3 \\
26 & 328.13 & -41.84 & 0.6 \\
\hline
\end{tabular}


Supplementary Table 3: FRB 170707 detections

\begin{tabular}{cccc} 
Beam & RA (J2000) & DEC (J2000) & S/N \\
& $(\mathrm{deg})$ & $(\mathrm{deg})$ & \\
\hline 00 & 44.17 & -56.58 & 5.2 \\
01 & 44.89 & -57.39 & 10.5 \\
02 & 45.64 & -58.19 & 3.4 \\
06 & 45.80 & -56.64 & 3.3 \\
07 & 46.56 & -57.44 & 2.7 \\
\hline
\end{tabular}

Supplementary Table 4: FRB 170712 detections

\begin{tabular}{cccc} 
Beam & RA (J2000) & DEC (J2000) & S/N \\
& $(\mathrm{deg})$ & $(\mathrm{deg})$ & \\
\hline 16 & 339.02 & -60.67 & 13.8 \\
09 & 337.18 & -60.66 & 0.5 \\
10 & 338.09 & -61.45 & 6.4 \\
15 & 338.11 & -59.89 & 2.4 \\
17 & 339.97 & -61.44 & 6.9 \\
21 & 339.91 & -59.88 & 0.7 \\
22 & 340.86 & -60.65 & 1.9 \\
\hline
\end{tabular}


Beam RA (J2000) DEC (J2000) S/N

\begin{tabular}{llll} 
& $(\mathrm{deg})$ & $(\mathrm{deg})$ & \\
\hline 20 & 329.77 & -19.80 & 18.6 \\
19 & 330.39 & -20.49 & 10.1 \\
14 & 330.71 & -19.64 & 8.6 \\
21 & 329.16 & -19.11 & 3.5 \\
26 & 329.44 & -20.64 & 7.4 \\
27 & 328.83 & -19.95 & 3.7 \\
\hline
\end{tabular}

Supplementary Table 6: FRB 171003 detections

\begin{tabular}{cccc}
\hline \hline Beam & RA (J2000) & DEC (J2000) & S/N \\
& $(\mathrm{deg})$ & $(\mathrm{deg})$ & \\
\hline 06 & 187.96 & -13.05 & 0.1 \\
18 & 186.74 & -14.06 & 8.1 \\
12 & 187.65 & -13.90 & 14.3 \\
13 & 187.05 & -13.22 & 3.4 \\
19 & 186.14 & -13.38 & 0.0 \\
25 & 185.82 & -14.22 & 0.0 \\
24 & 186.42 & -14.91 & 1.1 \\
\hline
\end{tabular}




\begin{tabular}{cccc} 
Beam & RA (J2000) & DEC (J2000) & S/N \\
& $(\mathrm{deg})$ & $(\mathrm{deg})$ & \\
\hline 26 & 179.29 & -12.07 & 11.4 \\
25 & 179.89 & -12.76 & 1.6 \\
31 & 178.98 & -12.91 & 1.4 \\
32 & 178.39 & -12.22 & 2.3 \\
27 & 178.71 & -11.38 & 3.1 \\
20 & 179.61 & -11.22 & 4.8 \\
19 & 180.20 & -11.91 & 4.7 \\
\hline
\end{tabular}

Supplementary Table 8: FRB 171019 detections

\begin{tabular}{cccc}
\hline \hline Beam & RA (J2000) & DEC (J2000) & S/N \\
& $(\mathrm{deg})$ & $(\mathrm{deg})$ & \\
\hline 21 & 334.59 & -8.34 & 24.3 \\
20 & 335.17 & -9.03 & 9.1 \\
15 & 335.48 & -8.18 & 0.5 \\
16 & 334.90 & -7.49 & 1.1 \\
22 & 334.00 & -7.65 & 4.4 \\
28 & 333.69 & -8.49 & 12.1 \\
27 & 334.27 & -9.18 & 17.9 \\
\hline
\end{tabular}


Supplementary Table 9: FRB 171020 detections

\begin{tabular}{cccc} 
Beam & RA (J2000) & DEC (J2000) & S/N \\
& $(\mathrm{deg})$ & $(\mathrm{deg})$ & \\
\hline 00 & 333.22 & -19.99 & 24.4 \\
01 & 332.59 & -19.31 & 4.0 \\
06 & 332.27 & -20.16 & 0.0 \\
07 & 331.65 & -19.48 & 1.8 \\
12 & 331.96 & -21.01 & 0.9 \\
\hline
\end{tabular}

Supplementary Table 10: FRB 171116 detections

\begin{tabular}{cccc} 
Beam & RA (J2000) & DEC (J2000) & S/N \\
& $(\mathrm{deg})$ & $(\mathrm{deg})$ & \\
\hline 28 & 53.34 & -17.35 & 11.9 \\
22 & 53.66 & -16.51 & 1.8 \\
21 & 54.27 & -17.20 & 0.0 \\
27 & 53.94 & -18.04 & 0.3 \\
33 & 53.00 & -18.19 & 4.6 \\
34 & 52.40 & -17.50 & 6.0 \\
29 & 52.74 & -16.65 & 8.1 \\
\hline
\end{tabular}




\begin{tabular}{cccc} 
Beam & RA (J2000) & DEC (J2000) & S/N \\
& $(\mathrm{deg})$ & $(\mathrm{deg})$ & \\
\hline 01 & 54.99 & -11.97 & 2.4 \\
02 & 54.39 & -11.28 & 27.1 \\
03 & 53.80 & -10.60 & 6.8 \\
07 & 54.08 & -12.13 & 2.0 \\
08 & 53.49 & -11.44 & 2.0 \\
\hline
\end{tabular}

Supplementary Table 12: FRB 171216 detections

\begin{tabular}{cccc}
\hline Beam & RA (J2000) & DEC (J2000) & S/N \\
& $(\mathrm{deg})$ & $(\mathrm{deg})$ & \\
\hline 20 & 49.91 & -57.47 & 0.6 \\
21 & 50.77 & -58.25 & 0.3 \\
26 & 50.72 & -56.69 & 1.5 \\
27 & 51.58 & -57.46 & 8.4 \\
28 & 52.48 & -58.22 & -0.6 \\
32 & 52.36 & -56.66 & 8.6 \\
33 & 53.25 & -57.42 & 3.4 \\
\hline
\end{tabular}




\begin{tabular}{cccc}
\hline \hline Beam & RA (J2000) & DEC (J2000) & S/N \\
& $(\mathrm{deg})$ & $(\mathrm{deg})$ & \\
\hline 30 & 327.44 & -34.30 & -0.1 \\
31 & 328.00 & -35.08 & 34.7 \\
32 & 328.57 & -35.85 & 30.6 \\
33 & 329.15 & -36.62 & 0.1 \\
25 & 326.90 & -35.08 & 2.2 \\
beam26 & 327.46 & -35.86 & 16.7 \\
\hline
\end{tabular}

\begin{tabular}{cccc}
\multicolumn{4}{c}{ Supplementary Table 14: FRB 180119 detections } \\
\hline \hline Beam & RA (J2000) & DEC (J2000) & S/N \\
& $(\mathrm{deg})$ & $(\mathrm{deg})$ & \\
\hline 19 & 52.86 & -13.14 & 9.1 \\
20 & 52.27 & -12.45 & 17.3 \\
21 & 51.68 & -11.76 & 2.0 \\
14 & 53.17 & -12.29 & 3.3 \\
15 & 52.58 & -11.60 & 2.3 \\
26 & 51.95 & -13.29 & 9.0 \\
27 & 51.36 & -12.60 & 2.7 \\
\hline
\end{tabular}




\begin{tabular}{cccc}
\multicolumn{4}{c}{ Supplementary Table 15: FRB 180128 detections } \\
\hline \hline Beam & RA (J2000) & DEC (J2000) & S/N \\
& $(\mathrm{deg})$ & $(\mathrm{deg})$ & \\
\hline 11 & 209.01 & -6.73 & 13.0 \\
05 & 209.90 & -6.58 & 3.4 \\
10 & 209.59 & -7.42 & 4.2 \\
17 & 208.70 & -7.58 & 1.7 \\
\hline
\end{tabular}

Supplementary Table 16: FRB 180128.2 detections

\begin{tabular}{cccc}
\hline Beam & RA (J2000) & DEC (J2000) & S/N \\
& $(\mathrm{deg})$ & $(\mathrm{deg})$ & \\
\hline 03 & 335.35 & -60.63 & 10.8 \\
02 & 334.53 & -59.83 & 6.6 \\
04 & 336.21 & -61.43 & -2.1 \\
08 & 336.32 & -59.87 & 7.3 \\
09 & 337.18 & -60.66 & 4.2 \\
\hline
\end{tabular}


Beam RA (J2000) DEC (J2000) S/N

\begin{tabular}{llll} 
& $(\mathrm{deg})$ & $(\mathrm{deg})$ & \\
\hline 22 & 328.05 & -38.19 & 10.8 \\
21 & 327.47 & -37.42 & -0.5 \\
23 & 328.65 & -38.97 & 6.7 \\
16 & 326.91 & -38.20 & 2.3 \\
17 & 327.49 & -38.98 & 7.0 \\
28 & 328.61 & -37.41 & 1.7 \\
29 & 329.20 & -38.18 & 1.8 \\
\hline
\end{tabular}

Supplementary Table 18: FRB 180131 detections

\begin{tabular}{cccc}
\hline Beam & RA (J2000) & DEC (J2000) & S/N \\
& $(\mathrm{deg})$ & $(\mathrm{deg})$ & \\
\hline 25 & 327.52 & -41.06 & 14.8 \\
24 & 326.92 & -40.28 & 9.3 \\
30 & 328.10 & -40.28 & 9.0 \\
31 & 328.71 & -41.05 & 1.9 \\
26 & 328.13 & -41.84 & -1.0 \\
19 & 326.92 & -41.84 & 0.2 \\
18 & 326.32 & -41.06 & 3.5 \\
\hline
\end{tabular}




\begin{tabular}{cccc}
\multicolumn{4}{c}{ Supplementary Table 19: FRB 180212 detections } \\
\hline \hline Beam & RA (J2000) & DEC (J2000) & S/N \\
& $(\mathrm{deg})$ & $(\mathrm{deg})$ & \\
\hline 05 & 215.27 & -3.80 & 18.3 \\
04 & 215.85 & -4.49 & 4.7 \\
10 & 214.96 & -4.64 & 0.9 \\
11 & 214.38 & -3.95 & 3.1 \\
\hline
\end{tabular}




\section{Supplementary Figures}
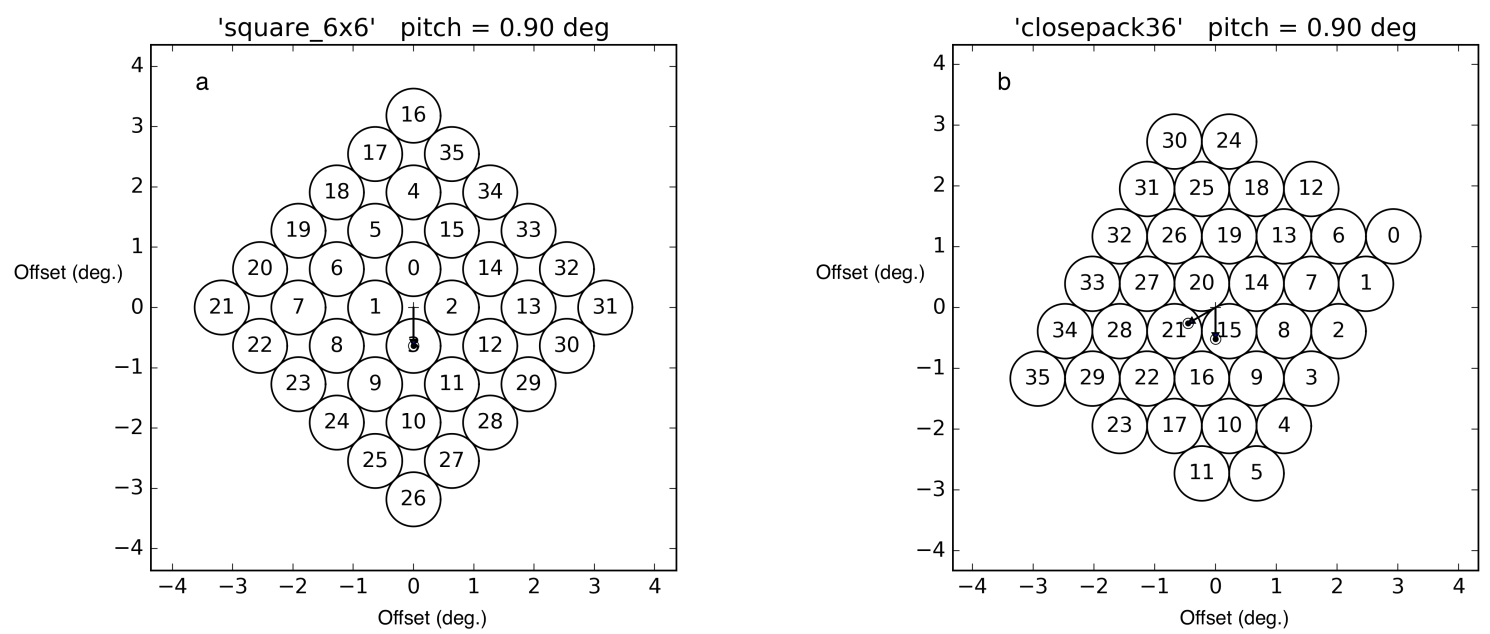

Supplementary Figure 1: Phase array beam configurations. In panel a, we show the square configuration used for initial survey that discovered FRB 170107. In panel b, we show the hexagonal configuration used in subsequent searches. The circle diameters are 0.9 degree, slightly smaller than the FWHP beam point at these frequencies ${ }^{6}$, which is $\approx 1.2 \operatorname{deg}(\nu / 1.3 \mathrm{GHz})^{-1}$.
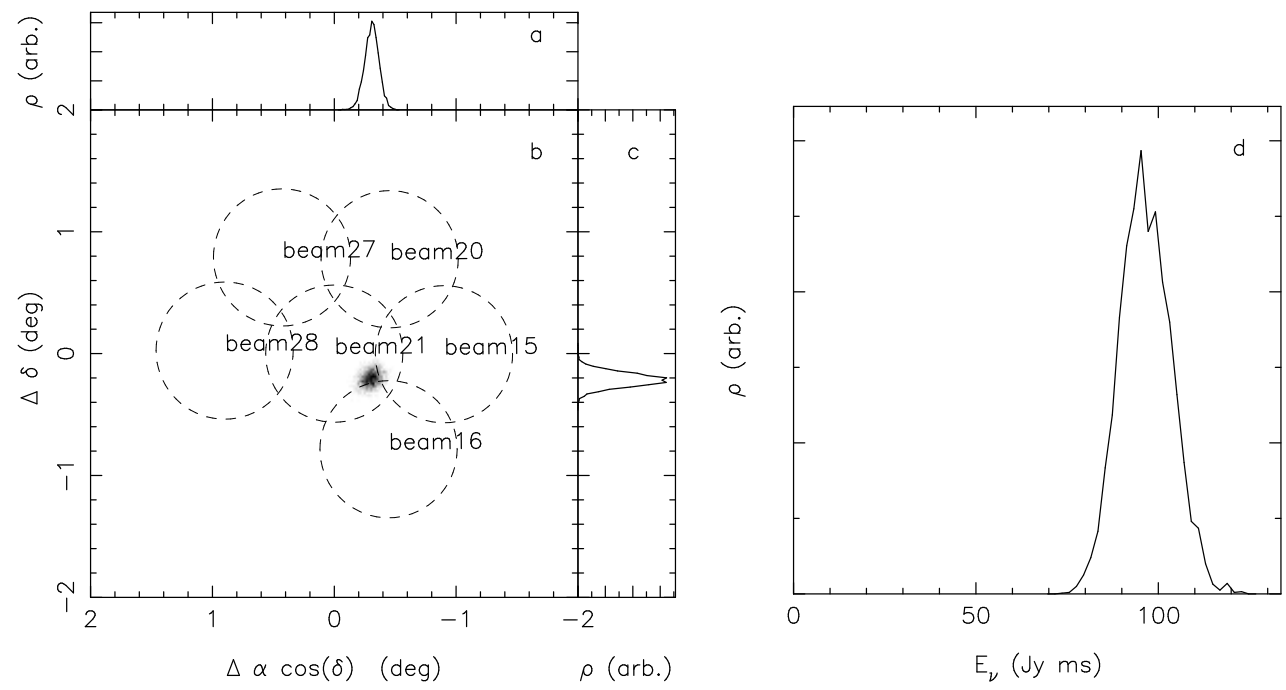

Supplementary Figure 2: Constraints on position and fluence for FRB 170416. Panels a, b and c show the one and two-dimensional posterior distributions for burst position. Panel d shows the constraint on burst fluence. 

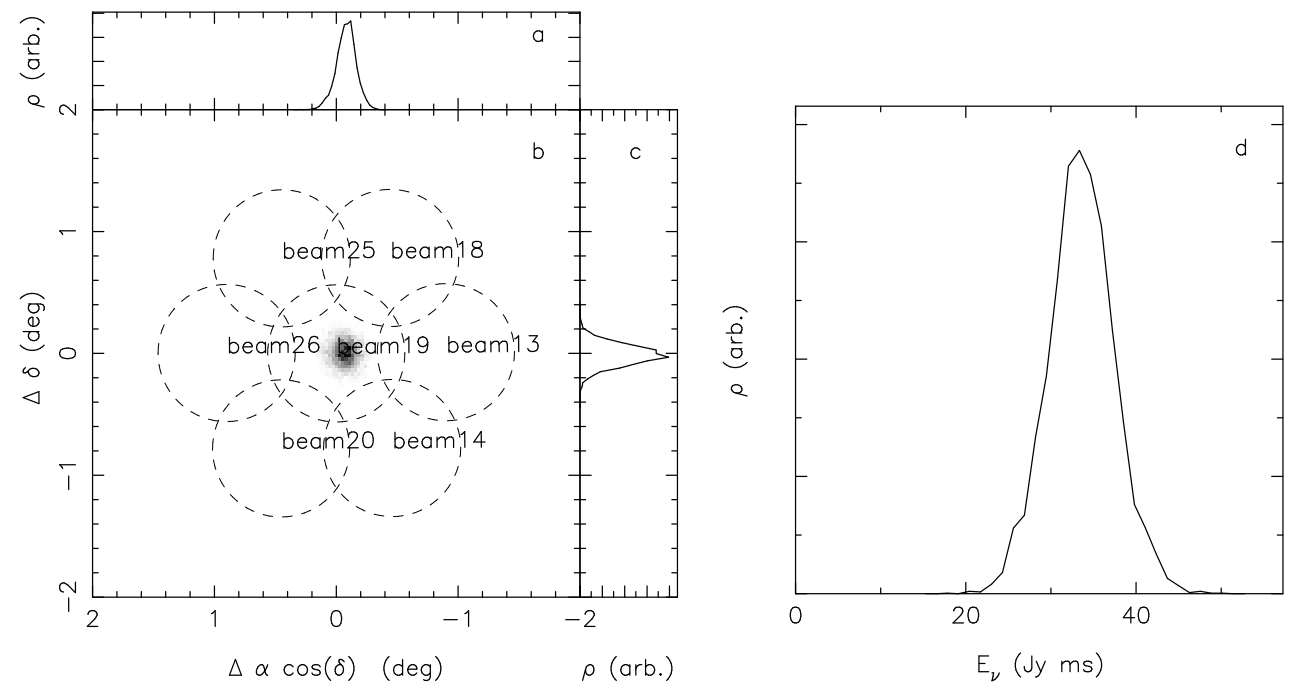

Supplementary Figure 3: Constraints on position and fluence for FRB 170428. Panels a, b and c show the one and two-dimensional posterior distributions for burst position. Panel d shows the constraint on burst fluence.
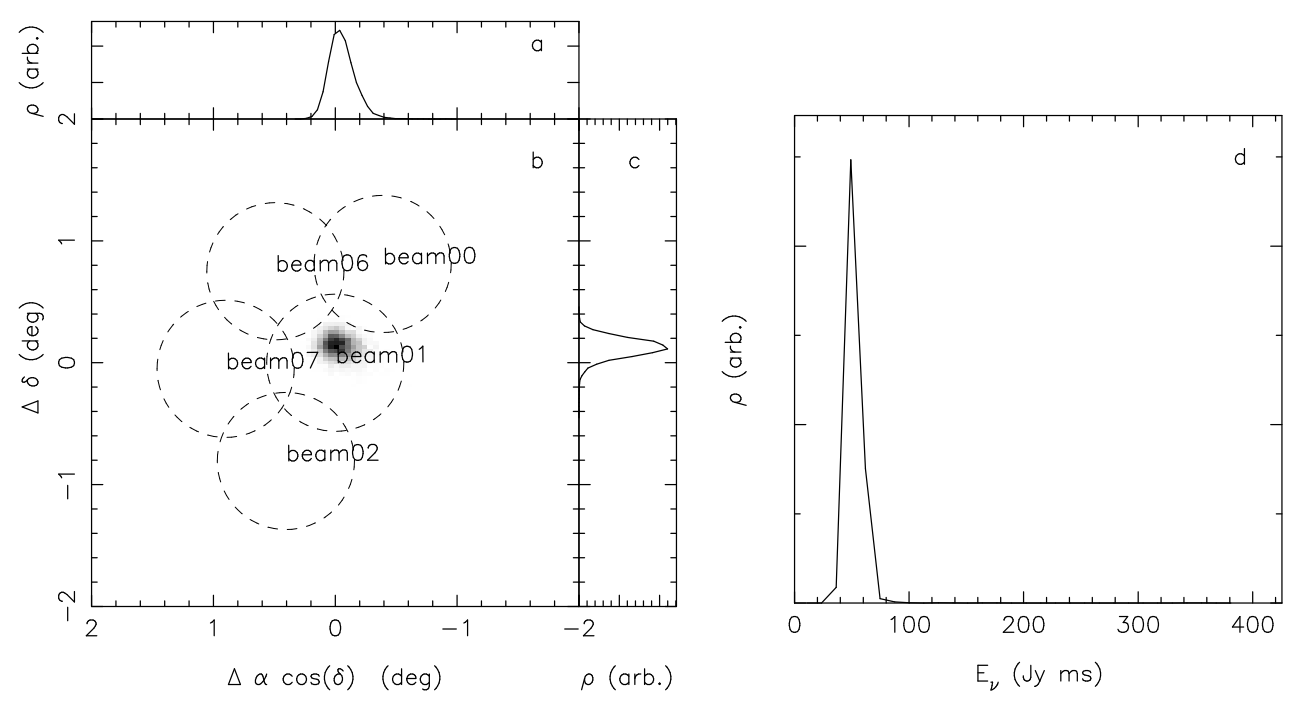

Supplementary Figure 4: Constraints on position and fluence for FRB 170707. Panels a, b and c show the one and two-dimensional posterior distributions for burst position. Panel d shows the constraint on burst fluence. 

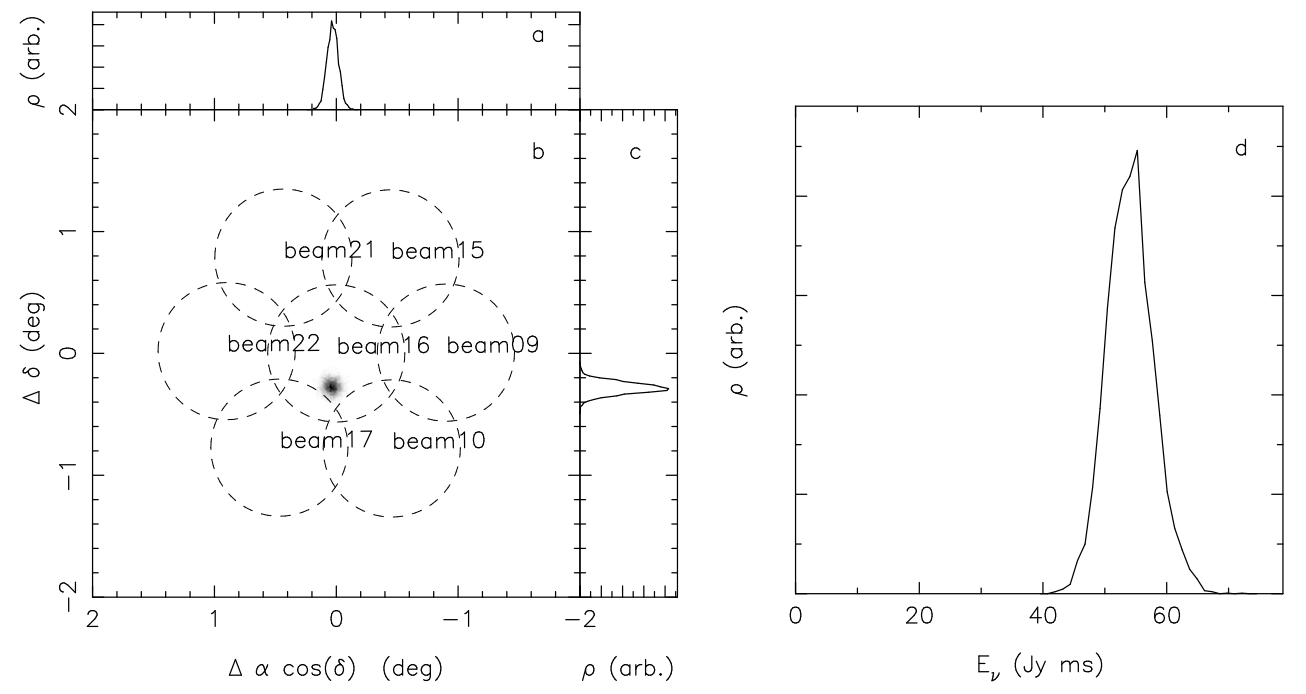

Supplementary Figure 5: Constraints on position and fluence for FRB 170712. Panels a, b and c show the one and two-dimensional posterior distributions for burst position. Panel d shows the constraint on burst fluence.
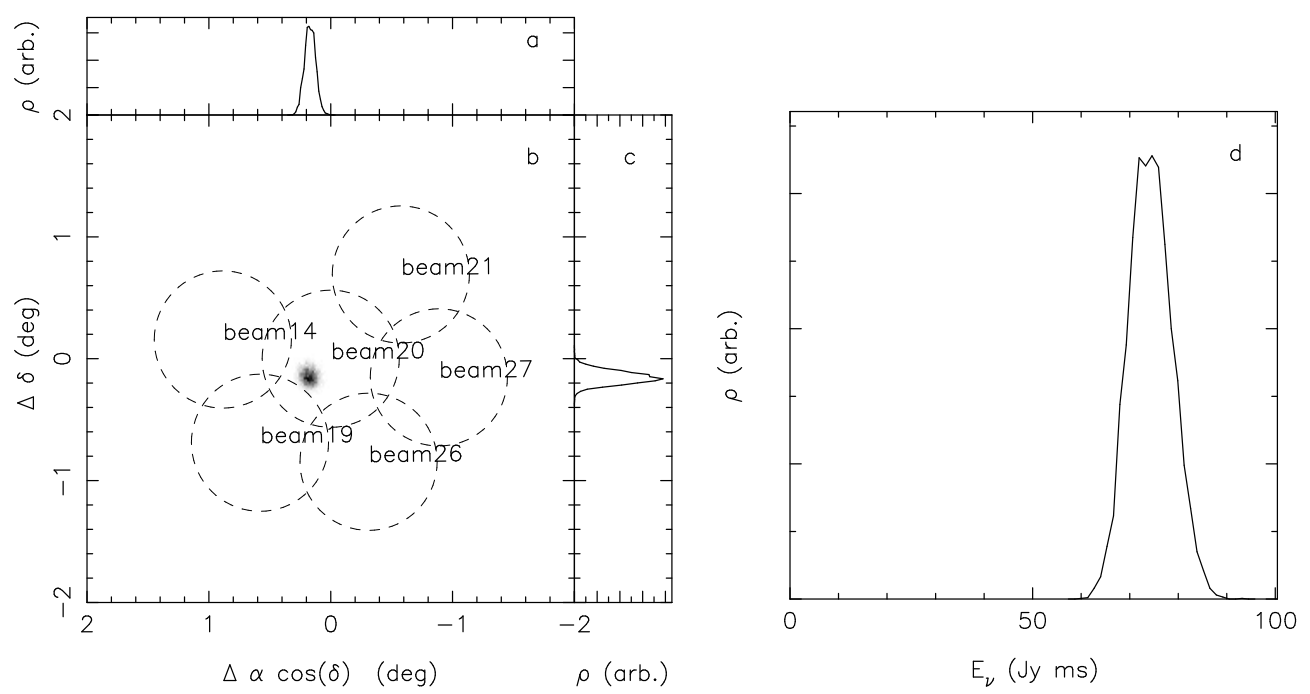

Supplementary Figure 6: Constraints on position and fluence for FRB 170906. Panels a, b and c show the one and two-dimensional posterior distributions for burst position. Panel d shows the constraint on burst fluence. 

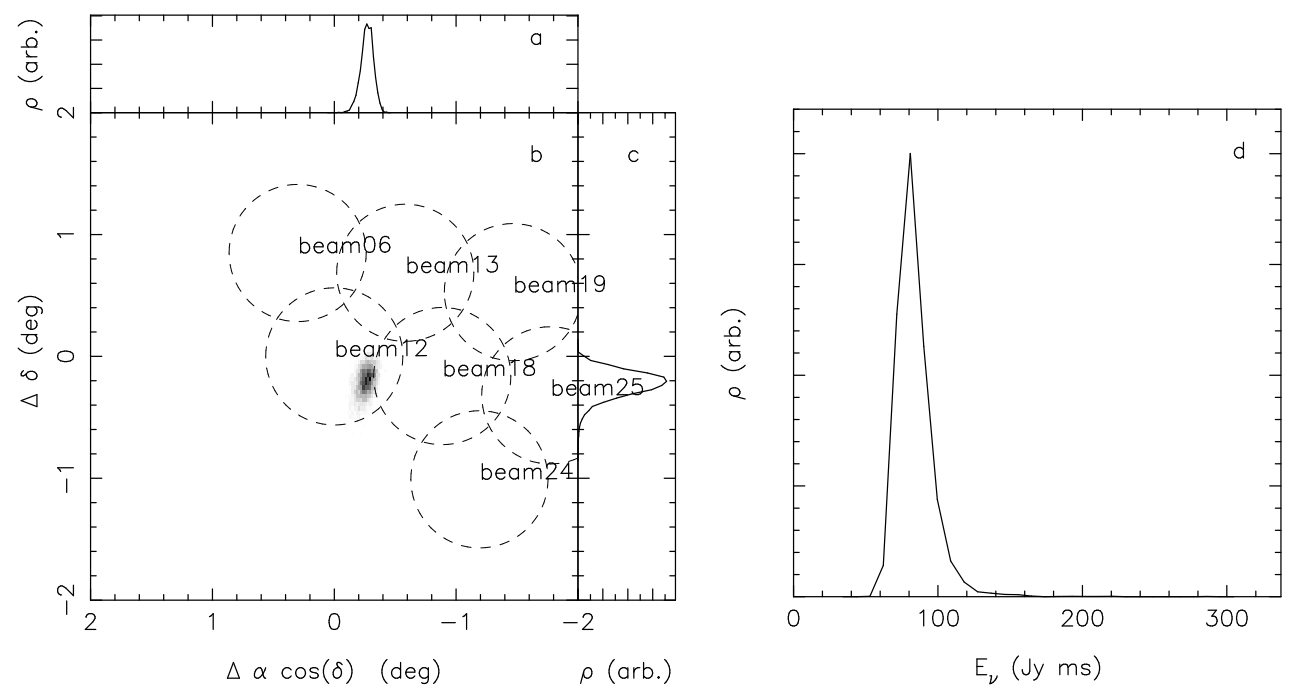

Supplementary Figure 7: Constraints on position and fluence for FRB 171003. Panels a, b and c show the one and two-dimensional posterior distributions for burst position. Panel d shows the constraint on burst fluence.
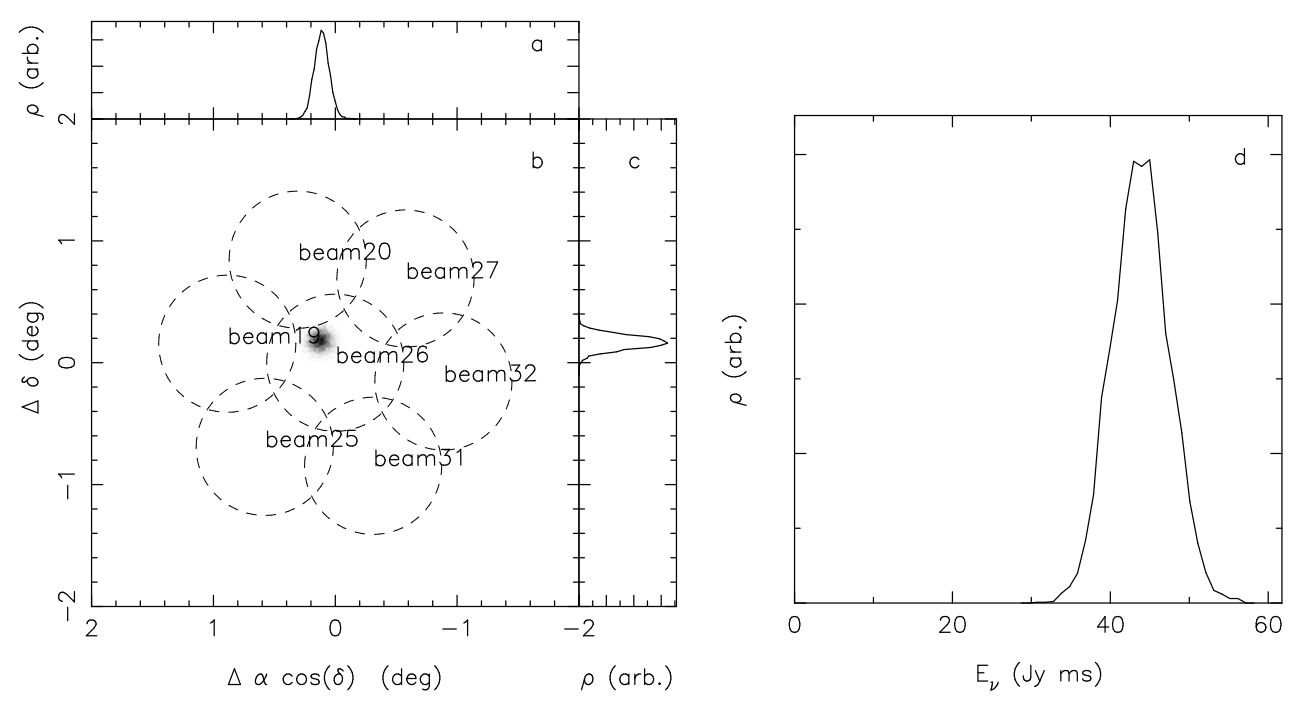

Supplementary Figure 8: Constraints on position and fluence for FRB 171004. Panels a, b and c show the one and two-dimensional posterior distributions for burst position. Panel d shows the constraint on burst fluence. 

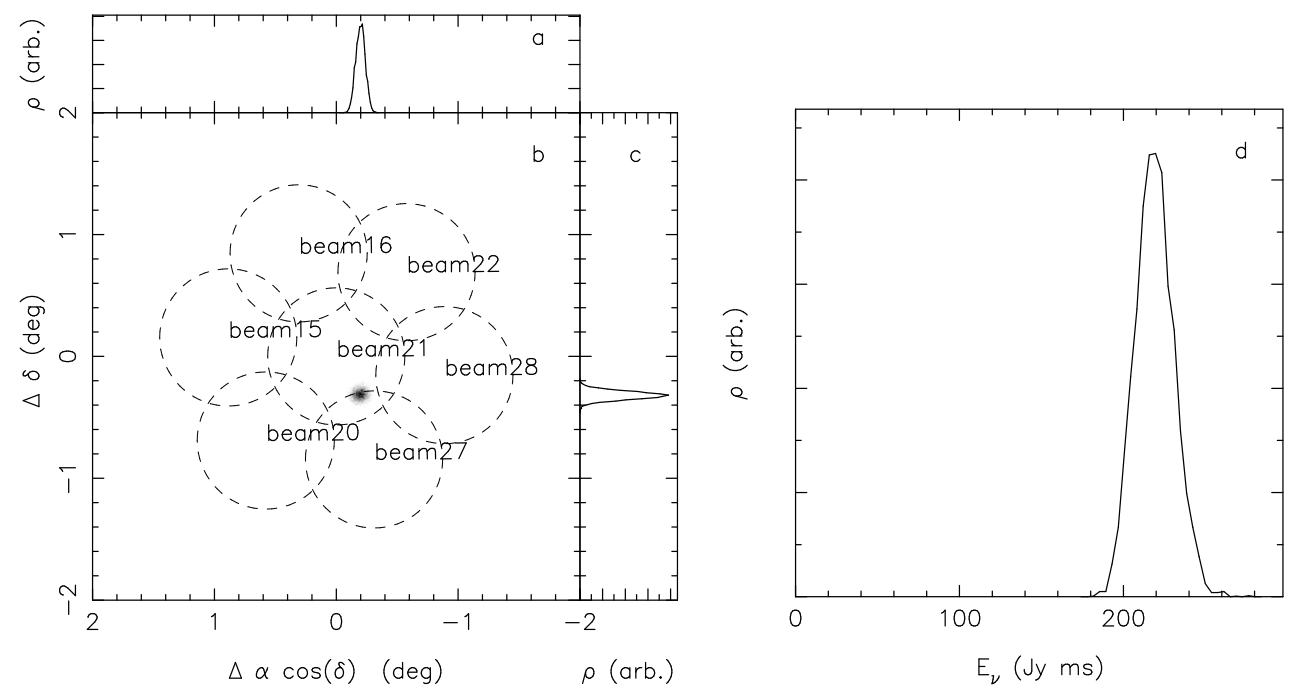

Supplementary Figure 9: Constraints on position and fluence for FRB 171019. Panels a, b and c show the one and two-dimensional posterior distributions for burst position. Panel d shows the constraint on burst fluence.
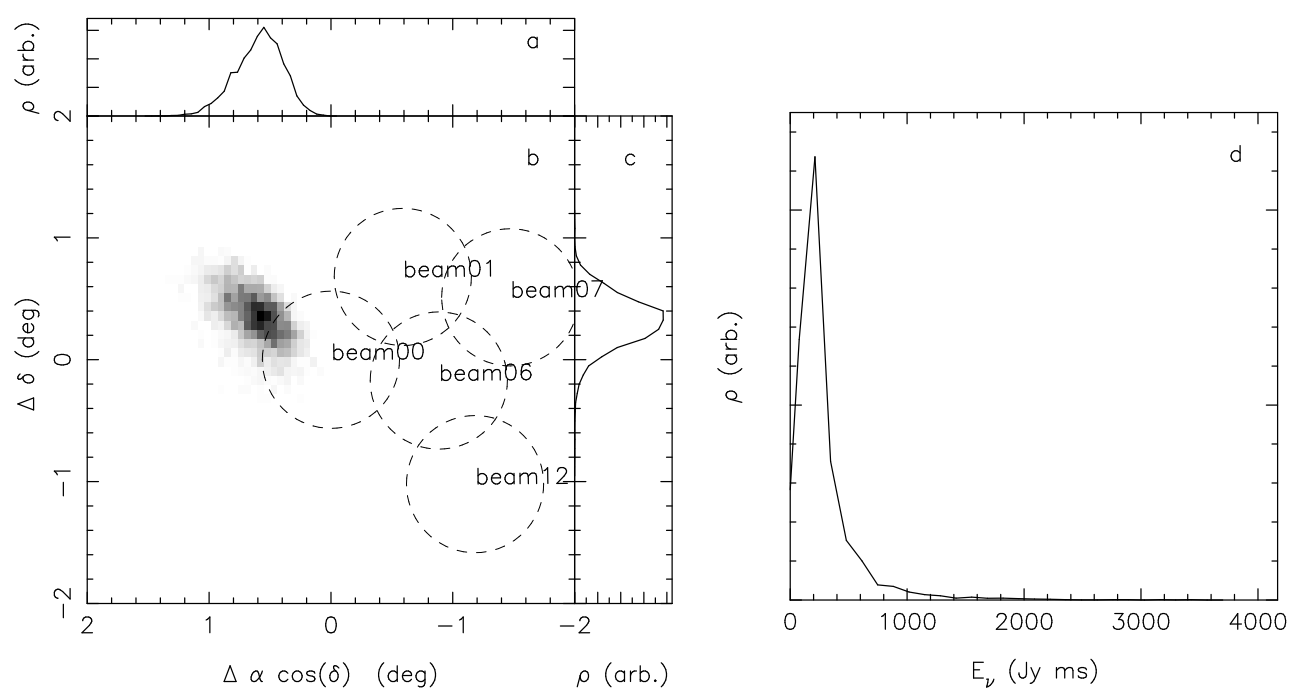

Supplementary Figure 10: Constraints on position and fluence for FRB 171020. Panels a, b and c show the one and two-dimensional posterior distributions for burst position. Panel d shows the constraint on burst fluence. 

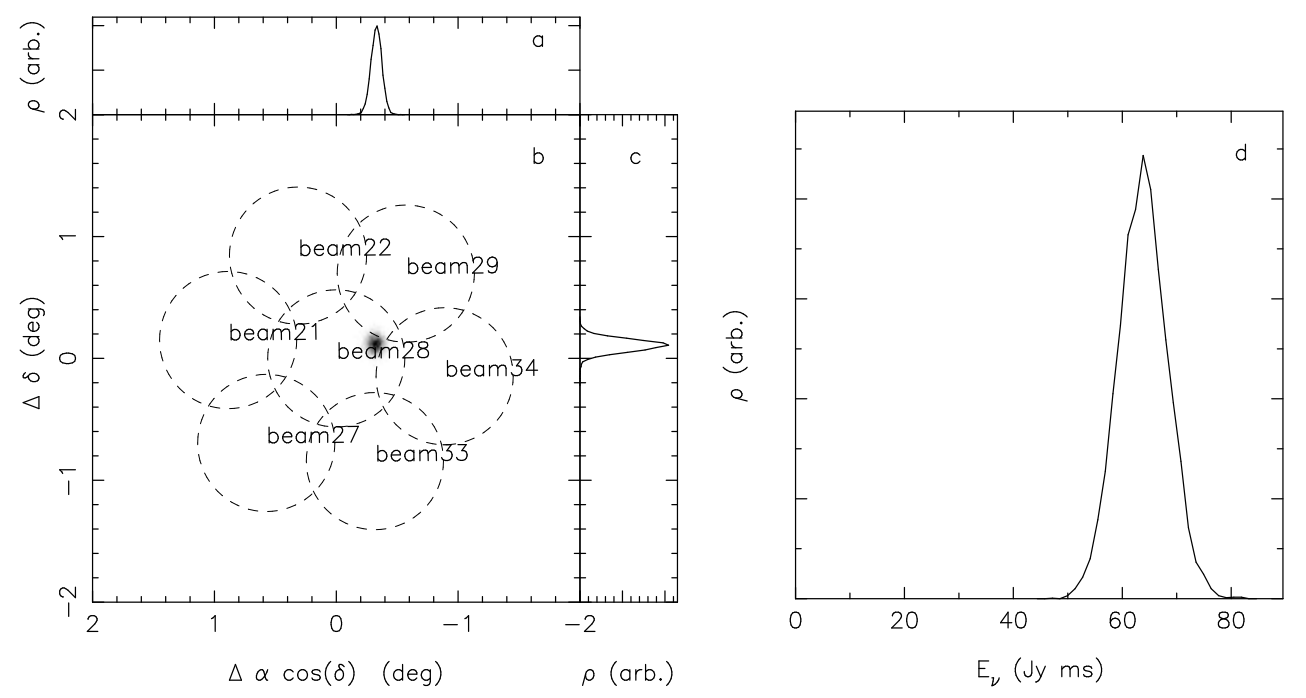

Supplementary Figure 11: Constraints on position and fluence for FRB 171116. Panels a, b and c show the one and two-dimensional posterior distributions for burst position. Panel d shows the constraint on burst fluence.
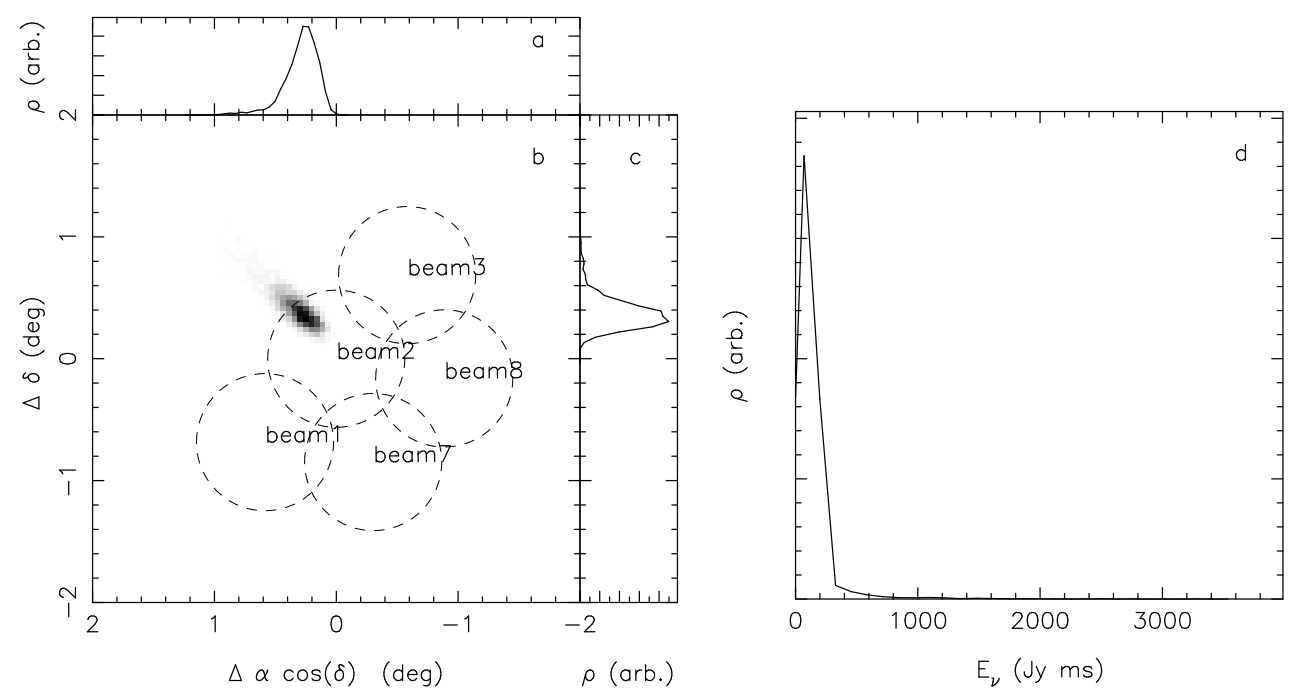

Supplementary Figure 12: Constraints on position and fluence for FRB 171213. Panels a, b and c show the one and two-dimensional posterior distributions for burst position. Panel d shows the constraint on burst fluence 

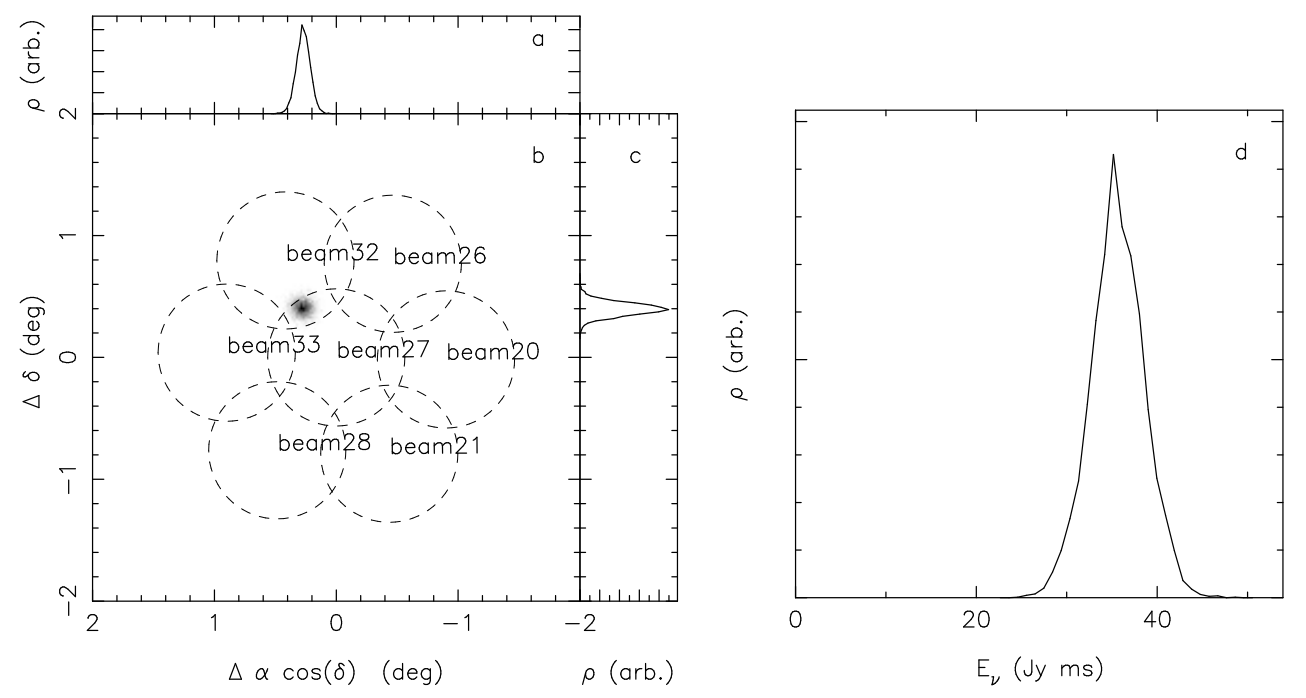

Supplementary Figure 13: Constraints on position and fluence for FRB 171216. Panels a, b and c show the one and two-dimensional posterior distributions for burst position. Panel d shows the constraint on burst fluence
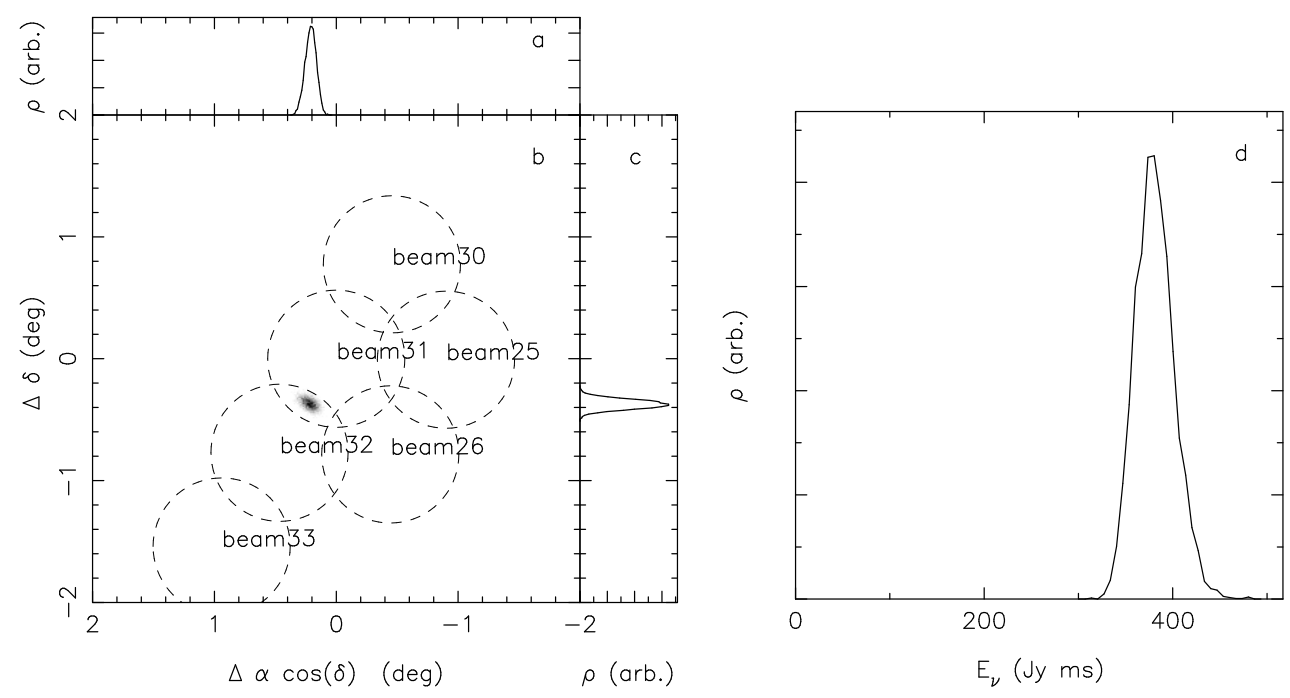

Supplementary Figure 14: Constraints on position and fluence for FRB 180110. Panels a, b and c show the one and two-dimensional posterior distributions for burst position. Panel d shows the constraint on burst fluence 

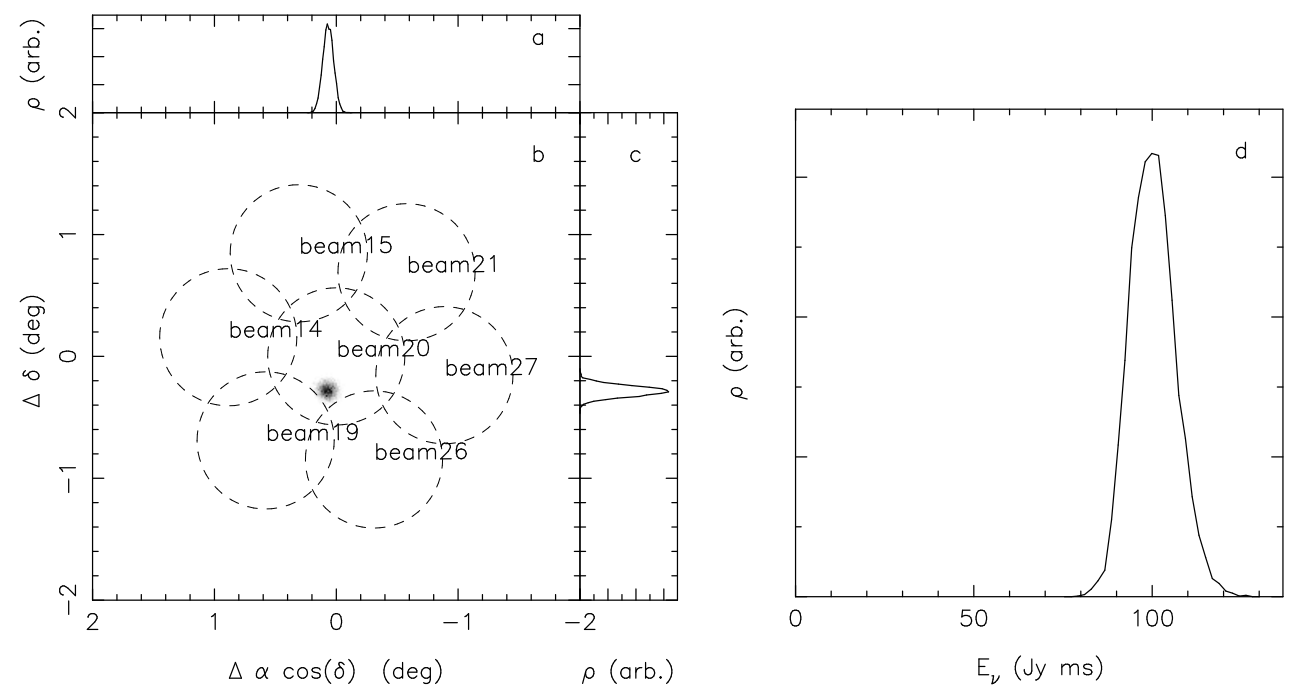

Supplementary Figure 15: Constraints on position and fluence for FRB 180119. Panels a, b and c show the one and two-dimensional posterior distributions for burst position. Panel d shows the constraint on burst fluence
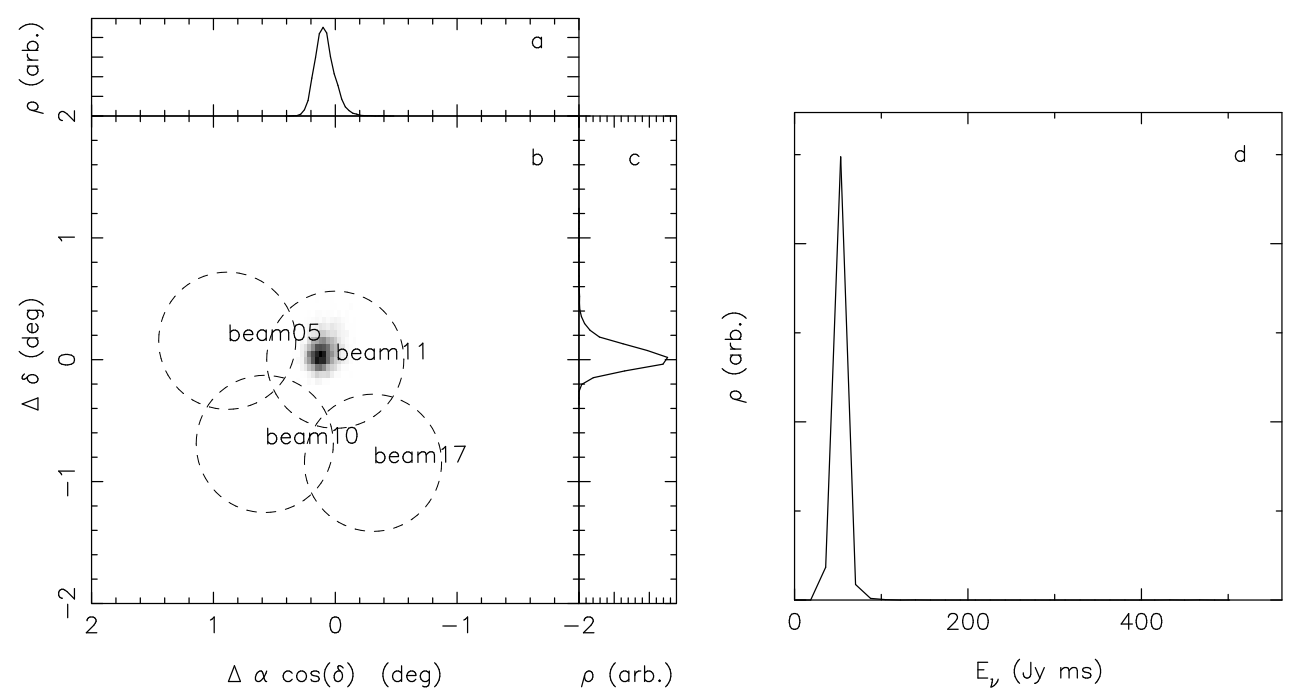

Supplementary Figure 16: Constraints on position and fluence for FRB 180128.0. Panels a, b and c show the one and two-dimensional posterior distributions for burst position. Panel d shows the constraint on burst fluence 

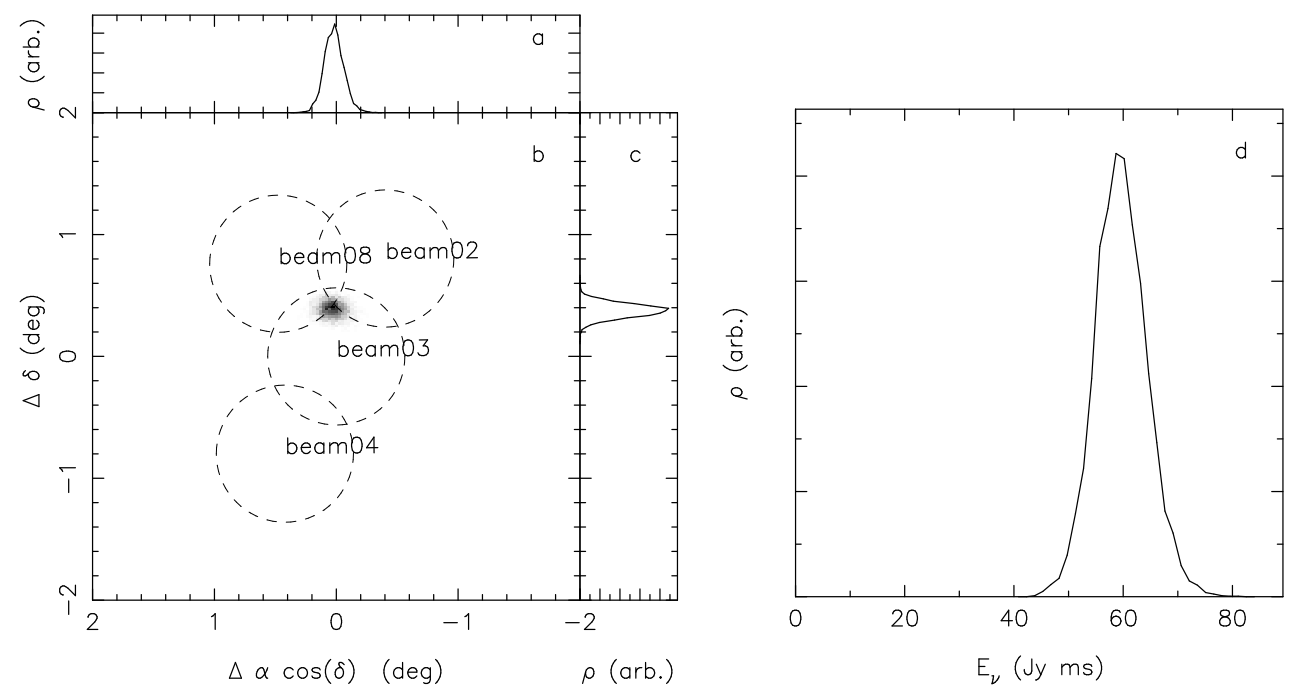

Supplementary Figure 17: Constraints on position and fluence for FRB 180128.2. Panels a, b and c show the one and two-dimensional posterior distributions for burst position. Panel d shows the constraint on burst fluence
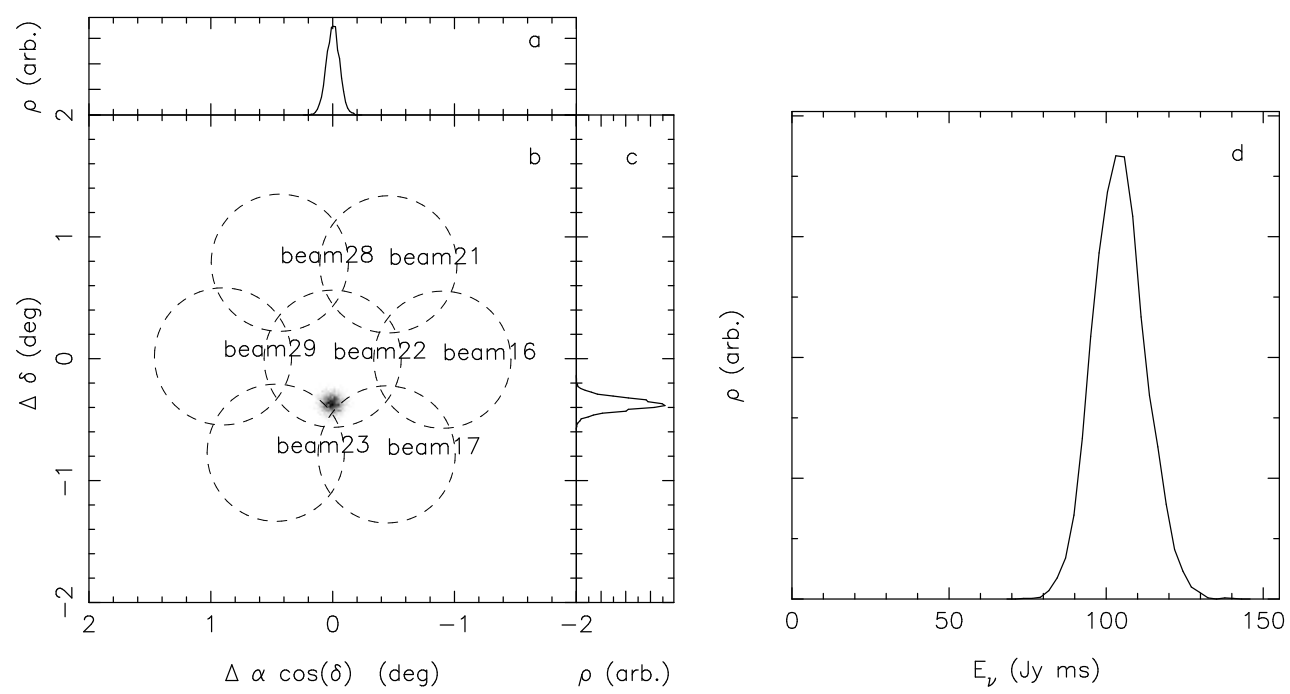

Supplementary Figure 18: Constraints on position and fluence for FRB 180130. Panels a, b and c show the one and two-dimensional posterior distributions for burst position. Panel d shows the constraint on burst fluence 

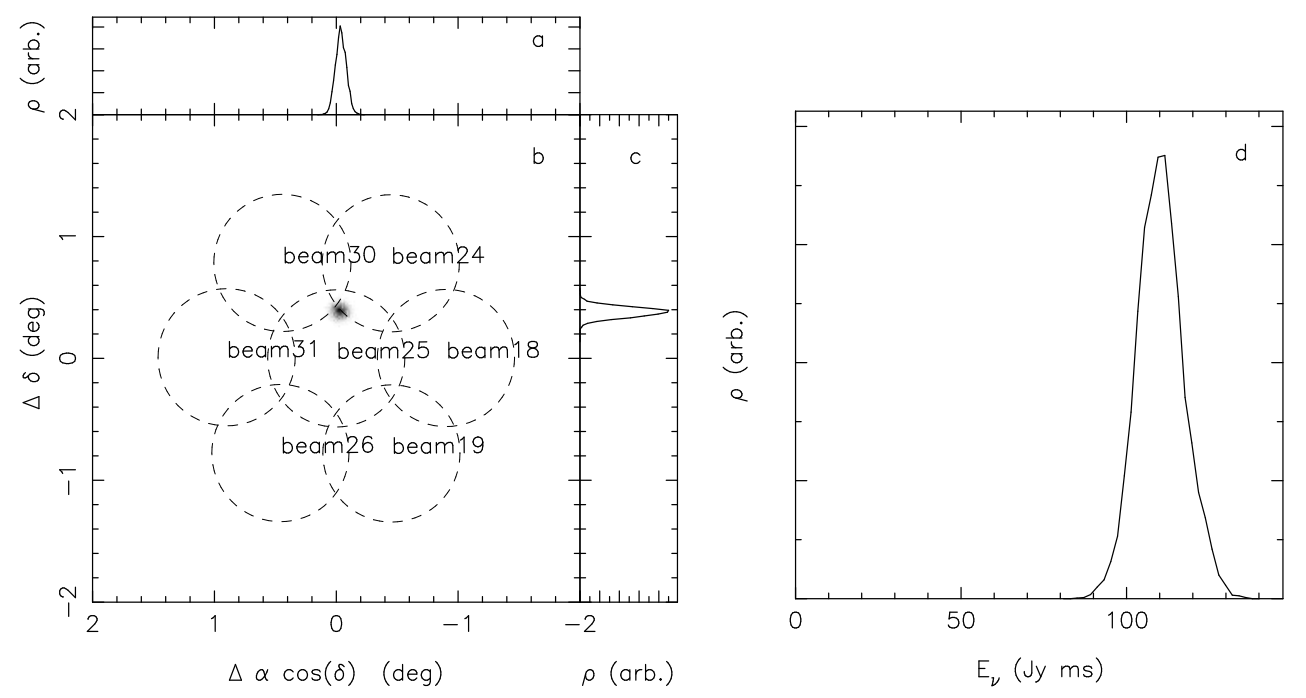

Supplementary Figure 19: Constraints on position and fluence for FRB 180131. Panels a, b and c show the one and two-dimensional posterior distributions for burst position. Panel $\mathrm{d}$ shows the constraint on burst fluence
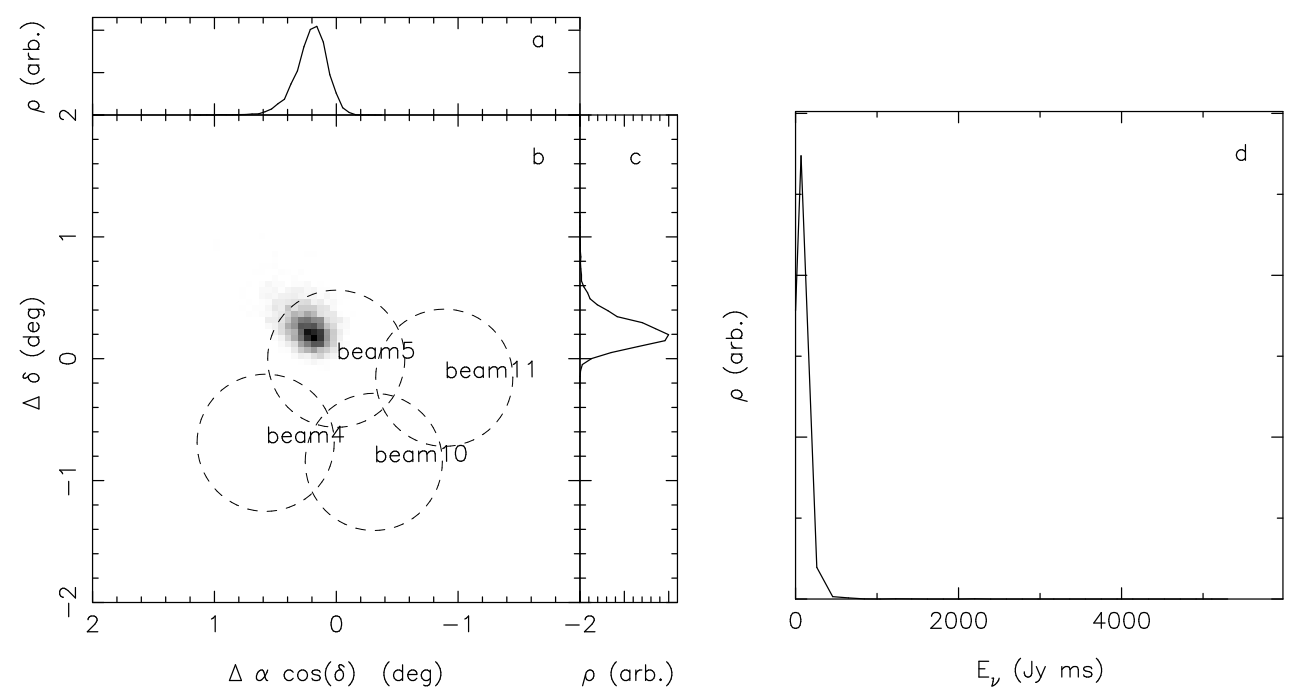

Supplementary Figure 20: Constraints on position and fluence for FRB 180212. Panels a, b and c show the one and two-dimensional posterior distributions for burst position. Panel d shows the constraint on burst fluence 


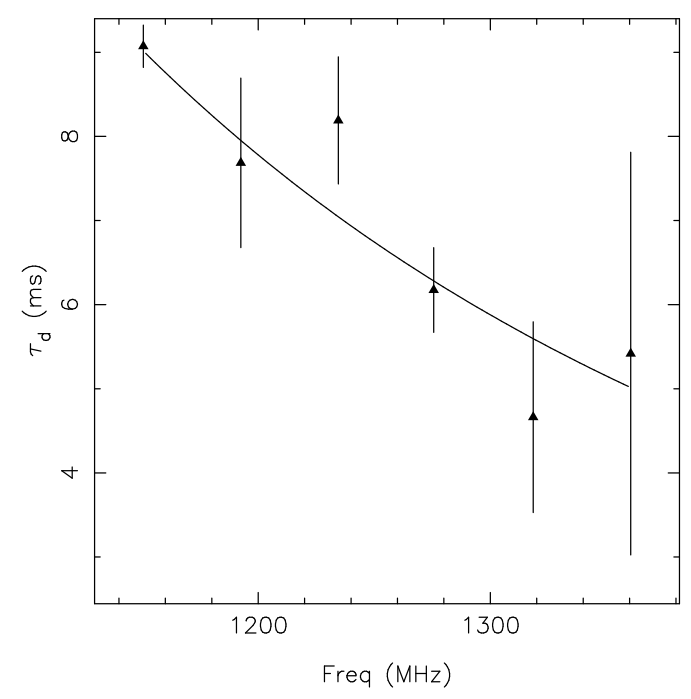

Supplementary Figure 21: Pulse broadening for FRB 180110. The broadening time $\tau_{d}$ shows significant change with frequency $\nu$. The solid line shows the best-fitting power law as a function of frequency $\tau_{d}(\nu)=(3.6 \pm$ $0.4 \mathrm{~ms})\left(\nu / \nu_{0}\right)^{-3.5 \pm 0.5}$, where the quoted uncertainties are $1 \sigma$. 

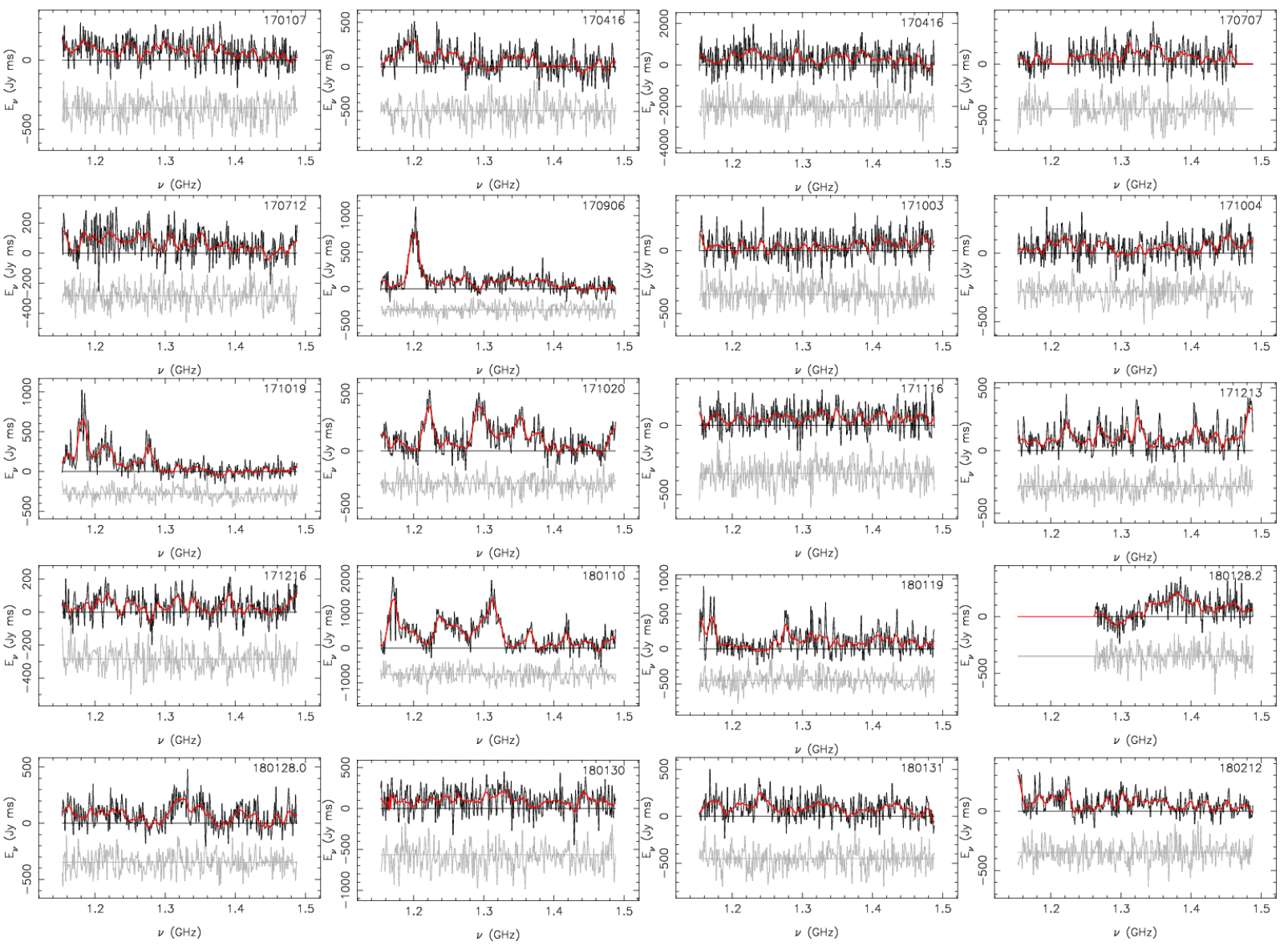

Supplementary Figure 22: Spectra for ASKAP FRBs. Spectra are produced from the primary beam detection, with the exception of FRB 171216, and have not been corrected for primary beam gain variations. For FRB 171216 the spectra has been produced by adding together the two $8 \sigma$ detections with equal weighting. Red lines are smoothed spectra, smoothed using a Gaussian Kernel with standard deviation of $4 \mathrm{MHz}$ ( 4 channels). Grey lines are example baseline spectra taken within $100 \mathrm{~ms}$ of the FRBs, and are offset from zero for clarity. Horizontal lines show zero power for both the on and off pulse spectra. For FRB 170707, a portion of the spectrum is missing due to spectral channels with zero beam weights (see supplementary text). For FRB 180128.2, the zeroed channels are flagged due to radio frequency interference (see supplementary text). 


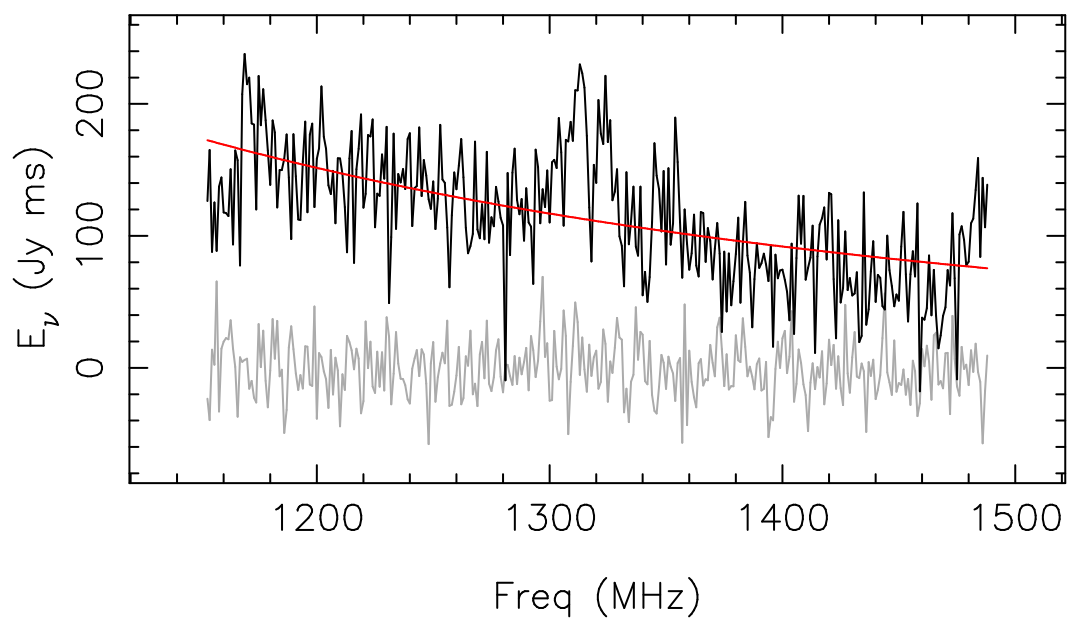

Supplementary Figure 23: Average spectrum of the ASKAP-detected FRBs. The spectrum is shown in black; in red, we show the best fitting power law (red). The gray spectrum shows the summed and dedispersed off-pulse spectra taken from within $100 \mathrm{~ms}$ of the FRB events. The average power-law spectra index is $-1.8 \pm 0.3$.

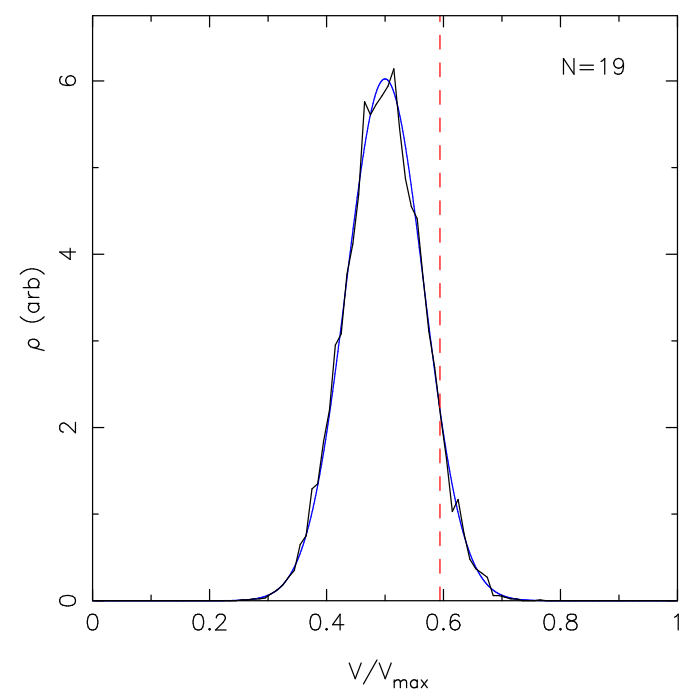

Supplementary Figure 24: Distribution of the $V / V_{\max }$ test statistic. The black line shows the simulated distribution. The blue curve is an exponential distribution with standard deviation $\sigma=1 / \sqrt{12 N}$, with $N=19$. The red dashed line is the value of test statistic derived from the 19 bursts detected above our completeness limit. 

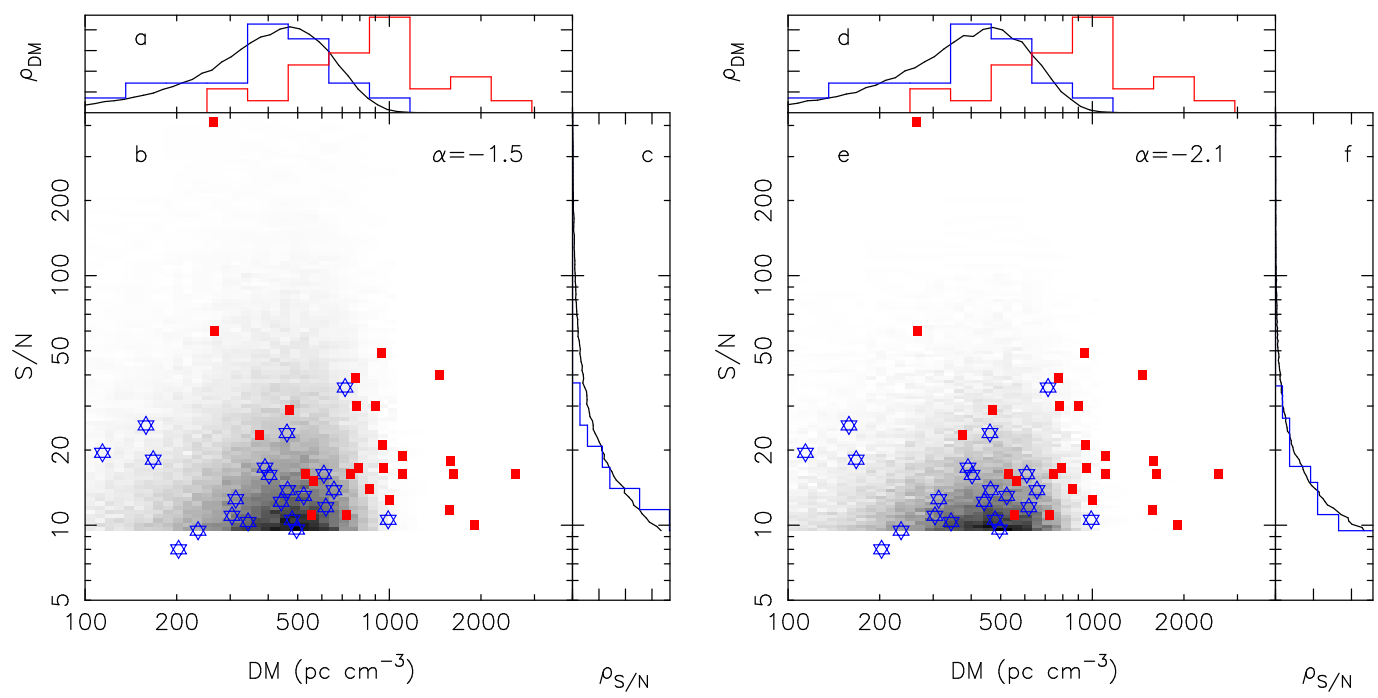

Supplementary Figure 25: Fluence-DM distributions for FRBs. In panels $a-c$ we show the simulated distributions for $\alpha=-1.5$. In panels $d-f$ we show distributions for and $\alpha=-2.1$ (right). Panels $a$ and $d$ : Dispersion-measure distribution. The black curve shows the simulated DM distribution. The blue histogram is the ASKAP DM distribution. The red histogram is the Parkes DM distribution. The simulations match the ASKAP DM distribution. Panels $b$ and $e$ : Joint distribution of S/N and DM. The grayscale shows the 2-D simulated distribution. The ASKAP FRBs are shown as blue stars. The Parkes FRBs are shown as red squares. Panels $c$ and $f: \mathrm{S} / \mathrm{N}$ distribution. The black curve shows the $\mathrm{S} / \mathrm{N}$ distribution from simulation. The blue curve shows the $\mathrm{S} / \mathrm{N}$ distribution from the ASKAP events. A Euclidean distribution produces an excess of bright events relative to what we observe. 


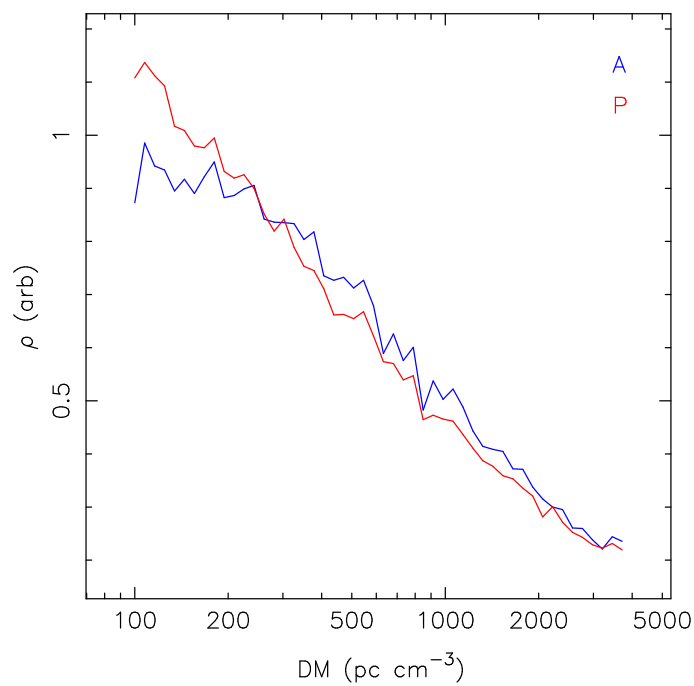

Supplementary Figure 26: Simulated response of receiving systems to dispersed pulses. The plot shows the response of ASKAP-like (blue) and Parkes-like (red) backend receiving systems to a power-law population of FRBs with a Euclidean fluence distribution. If the systems were ideal, (infinite time and frequency resolution) the distributions would be flat. At high DM $\left(>800 \mathrm{pc} \mathrm{cm}^{-3}\right)$ there is no difference in the shape of the distributions, indicating that the observed difference in median DM is not the result of the poorer time and frequency resolution of the ASKAP data-recording instrument. 


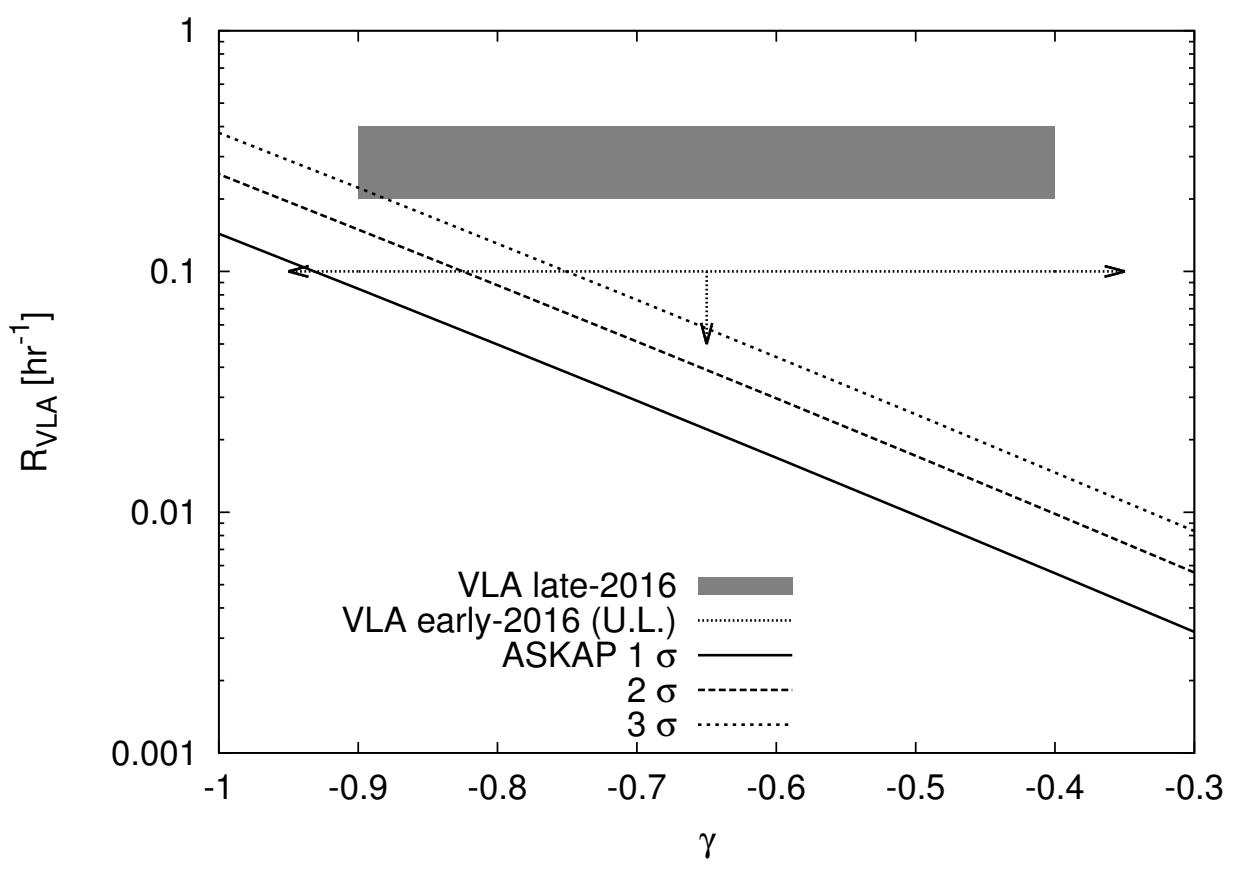

Supplementary Figure 27: Comparison of repetition of FRB 121102 to limits from ASKAP. The plot shows the limits on the repetition rate $R$ of ASKAP-detected FRBs at $184 \mathrm{mJy}$ ms sensitivity as a function of the cumulative repetition rate index $\alpha$, compared to observations of FRB 121102 by the JVLA ${ }^{26}$. 


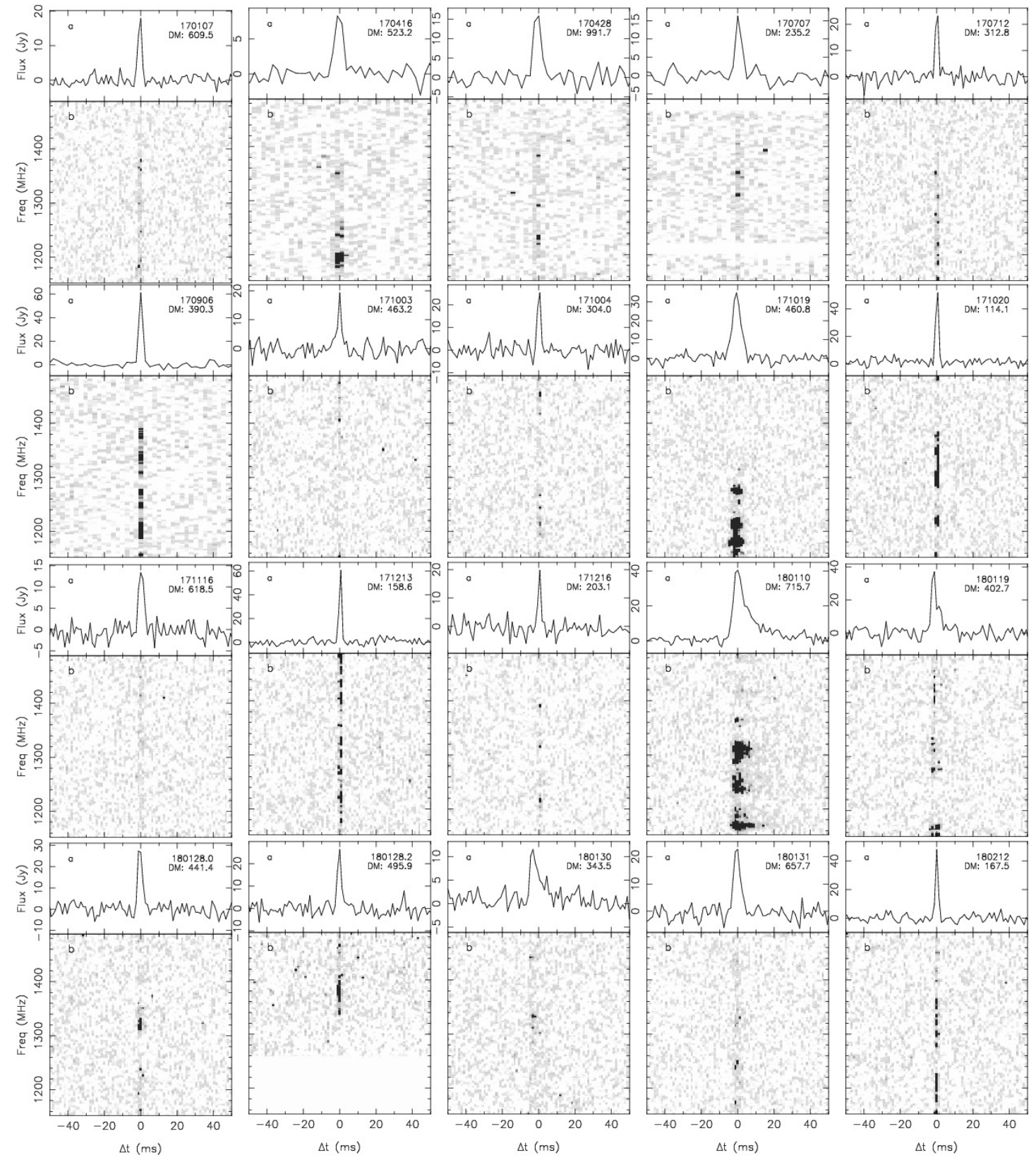

Supplementary Figure 28: Pulse profiles and dynamic spectra of ASKAP fast radio bursts. The FRB names and dispersion measures (DM) in units of $\mathrm{pc} \mathrm{cm}^{-3}$ are listed in the panels showing the pulse profiles (Panels a). The spectra (Panels b) have been dedispersed to the maximum-likelihood dispersion measure. The colour scale is set to range from the mean to $4 \sigma$ of the off-pulse intensity. Time averaged spectra can be found in Supplementary

Figure ??. 